\title{
An Immune Related Gene Signature from Chemokines and Chemokine Receptors to Predict Tumor Prognosis and Immune Microenvironment Alteration in Lower-Grade Glioma (LGG) Based on Large-Scale Transcriptome Profile Analyses
}

Chunxiao Qi

The First Hospital of China Medical University

Lei Lei

Dalian Municipal Central Hospital Affiliated of Dalian Medical University

Ningning Li

Dalian Municipal Central Hospital Affiliated of Dalian Medical University

Shaowu Ou ( $\nabla$ cmuoushaowu@163.com)

The First hospital of China Medical University https://orcid.org/0000-0002-9446-9576

\section{Research Article}

Keywords: glioma, immune microenvironment, chemokines, chemokine receptors

Posted Date: January 17th, 2022

DOI: https://doi.org/10.21203/rs.3.rs-1264057/v1

License: (c) (i) This work is licensed under a Creative Commons Attribution 4.0 International License. Read Full License 


\section{Abstract}

Chemokines and receptors are well known for their roles in mediating immune cell trafficking and lymphoid development; however, whether they can determine prognosis and immune microenvironment alterations in lower-grade glioma (LGG) remains uncertain. In this study, we acquired transcriptomic data from the Cancer Genome Atlas (TCGA), Chinese Glioma Genome Atlas (CGGA) and Gene Expression Omnibus (GEO) databases using a series of bioinformatics methods to estimate the prognostic value and potential biological function of candidate genes. We identified a four-gene (CCR4, CX3CL1, CXCL10 and CXCL11) signature to compose a risk score model. Kaplan-Meier curves and ROC curves verified the performance of risk score model in the TCGA, CGGA and GSE16011 datasets. The prognostic signature showed fair accuracy for 1-, 3- and 5-year overall survival (OS). The TIMER, TIP and CIBERSORT algorithms revealed different immune cell infiltration levels between the high- and low-risk score groups. LGG patients with high-risk scores had more malignant phenotypes and higher immune checkpoints expression as well as presented immunotherapeutic resistance. Therefore, we comprehensively analyzed the important role of chemokines and receptors in LGG patients; created a novel immune risk score model that could effectively reflect the prognosis and immune microenvironment alteration and presented a valuable indicator for immunotherapy in LGG patients.

\section{Introduction}

Gliomas are the most common primary brain tumors with aggressive growth features and treatment resistance. Glioblastoma (GBM, World Health Organization (WHO) grade IV) has the worst prognosis with 5\%-10\% of 5-year survival rates after diagnosis (Omuro et al. 2018). By comparison, patients with lower-grade glioma (LGG) (WHO grade II and III glioma) tend to have better prognoses, but the disease still has a significant impact on the patients' quality of life (Brat et al. 2015). Maximum resection to a safe degree with postoperative chemotherapy and radiotherapy has been considered a standard treatment strategy for newly diagnosed glioma patients (Stupp et al. 2005). However, mounting evidence suggests that considerable LGG patients inevitably recur and eventually progress to GBM (Cheng et al. 2019; Molenaar et al. 2014; Zhang et al. 2015). Despite decades of investigation into surgical techniques and chemotherapy medicine, there is still limited progress in the choice of treatment regimens, and the optimal treatment of LGG remains controversial. Accumulating evidence indicates that the tumor microenvironment (TME) performs better in predicting glioma prognosis and evaluating therapeutic efficiency (Jia et al. 2018; Lin et al. 2020). The TME mainly consists of cellular components and extracellular matrix, which constitute the heterogeneous features of tumors and account for immune resistance (Osipov et al. 2019; Wu and Dai 2017). Immune microenvironment alterations in tumor are usually accompanied by immunotherapeutic resistance and lead to a worse prognosis. The successful use of cytotoxic T-lymphocyte-associated antigen 4 (CTLA-4) and programmed death-1 (PD-1) immune checkpoint inhibitors in patients with melanoma and non-small cell lung carcinoma has encouraged us to focus on immunotherapies in glioma (Hodi et al. 2010; Rizvi et al. 2015). Therefore, the identification of immune-related gene markers and understanding of immune microenvironment alterations remain urgent for glioma treatment.

Chemokines are the largest subcategory of cytokines and can be subdivided into four main classes depending on the location of the first two cysteine (C) residues in their protein sequence: namely, CC-chemokines, CXC-chemokines, Cchemokines and CX3C-chemokines, provide chemotactic signals for immune cell trafficking to specific destination (Groblewska et al. 2020; Nagarsheth et al. 2017). Mounting evidence has indicated that chemokines and chemokine receptors play important roles in cancer processes, such as angiogenesis, tumor growth, proliferation and metastasis (Nagarsheth, et al. 2017). Based on the presence of a glutamic-leucine-arginine (ELR) motif at the N-terminal, CXC chemokines can be subdivided into ELR ${ }^{+}$chemokines with angiogenic features and ELR ${ }^{-}$chemokines with angiostatic effects (Mollica Poeta et al. 2019). CCL11, CCL2, CCL16, CCL18 and CXCL8 promote tumor angiogenesis and endothelial cell survival (Mollica et al. 2019). Chemokines, through binding with chemokine receptors expressed by tumor cells, directly promote cancer cell proliferation by activating PI3K/AKT/NF-kB and MAPK/ERK pathways (Balkwill 2004; Mollica et al. 2019). Chemokines and chemokine receptors are involved in cancer metastasis in several cancer types. The CCR10/CCL27 
axis facilitates the adhesion and survival of cells during metastatic spreading (Ben-Baruch. 2008), and CCL28 promotes breast cancer growth and metastasis spreading through MAPK/ERK pathway (Yang et al. 2017).

Targeting chemokines and chemokine receptors can play anticancer activities in many cancers (Mollica et al. 2019). The CCR2 inhibitor PF-04136309 reduced the number of tumor-associated macrophages (TAMs) and exerts a modest effect on tumor growth when used alone, while it acts synergistically with the chemotherapeutic agent Gemcitabine in pancreatic cancers (Mitchem et al. 2013). Affi 5, a CCR4 blocking monoclonal antibody, reduces tumor growth to affect the phenotype of myeloid cells and increases the number of infiltrating NK cells in a preclinical model of renal cancers. CCR4 is now considered a target for renal carcinoma patients (Berlato et al. 2017). CXCR4 inhibitors have strong antitumor and antimetastatic effects in solid tumors; ADM3645 reduced the growth of xenografts of GBM and medulloblastoma cell lines, and the CXCR4 antagonist PRX177561 increased the antitumor effects of bevacizumab and sunitinib in subcutaneous or orthotopic xenografts of GBM models (Gravina et al. 2017; Yang et al. 2007). Hence, chemokines and chemokine receptors are expected to become new therapies for cancers. However, the potential roles of chemokines and chemokine receptors in the modification of immune infiltration remain uncertain, and whether selectively targeting chemokines and chemokine receptors could complement and increase the efficacy of the immunotherapies that are currently being used in cancer treatment and effectively reflect the immune process and prognosis of LGG patients have not been explored.

In the present study, we first used the TISIDB database (http://cis.hku.hk/TISIDB), a user-friendly web portal integrated multiple types of data resources in oncoimmunology, to acquire gene list of chemokines and chemokine receptors (Ru et al. 2019). Next, a series of bioinformatics analyses were performed, and we created a novel four-gene risk score model from chemokines and chemokine receptors. The prognostic value and underlying immune-related function were explored by different independent datasets. We used three algorithms (TIMER, TIP and CIBERSORT) to calculate immune cell infiltration levels between high- and low-risk score groups (Li et al. 2020; Newman et al. 2015; Xu et al. 2018). By Tumor Immune Dysfunction and Exclusion (TIDE) and Immune Cell Abundance Identifier (ImmuCellAI)(Jiang et al. 2018; Miao et al. 2020), we estimated the immunotherapeutic effects for LGG patients, and a receiver operator characteristic (ROC) curve was used to assess the accuracy of our risk score model in immunotherapeutic evaluation. In conclusion, we comprehensively analyzed the prognostic roles of chemokines and chemokine receptors in LGG patients and constructed a novel immunerelated signature that showed valuable clinical effects.

\section{Materials And Methods}

\section{Data Collection and Processing}

The gene list of chemokines and chemokine receptors was acquired from the TISIDB database (Immune-related genes (chemokine and receptor))(http://cis.hku.hk/TISIDB)(Ru et al, 2019), and a total of 59 genes were obtained. The mRNA expression value (log2(norm_count+1)) of 58 chemokines and chemokine receptors (CCL18 was not included in this dataset) was obtained from a RNA-seq data of 530 LGG samples from The Cancer Genome Atlas (TCGA) database using the UCSC Xena browser (https://xenabrowser.net/datapages/). We removed the genes with $50 \%$ of samples having an mRNA relative expression value equal to 0 , and 46 genes were identified for further study. Another two RNA-seq datasets of diffuse glioma were acquired from the Chinese Glioma Genome Atlas (CGGA) database (www.cgga.org.cn), one containing 325 diffuse gliomas (dataset ID: mRNA-325; Platform: Illumina Hiseq 2000 or 2500. 182 LGGs (WHO II, n=103, WHO III, $\mathrm{n}=79$ ), 139 cases glioblastomas (GBMs) and 4 cases without WHO grade information) (Bao et al. 2014; Zhao et al. 2017), and another dataset containing 693 cases of diffuse glioma (dataset ID: mRNA-693; Platform: Illumina Hiseq. 443 LGGs (WHO II, n=188; WHO III, n=255) and 249 GBMs, and one case without defined WHO grade) (Liu et al. 2018; Wang et al. 2015). An mRNA microarray dataset contains 301 glioma samples were obtained from the CGGA database (dataset ID: mRNA-array_301; Platform: Agilent Whole Human Genome (Array); WHO grade II, n=115; WHO III, n=57; WHO grade IV, n=124; undefined WHO grade, n=3)(Fang et al. 2017, Zhao, et al, 2017). The GSE16011 dataset (Platform: Affymetrix GeneChip Human Genome U133 Plus 2.0 Array) was obtained from the Gene Expression Omnibus (GEO) database 
(https://www.ncbi.nlm.nih.gov/geo/)(Gravendeel et al. 2009), which contains 110 LGGs (WHO II and WHO III). The main clinical information in this study included overall survival (OS) time, survival status, IDH-1 status, 1p19q codeletion status, sex and age. Only LGG (WHO grade II and III) patients with an OS of more than 30 days were included. GBM patients and cases without a detail OS time or with an OS time less than 30 days were removed. Finally, 1346 LGG patients were included in this study (TCGA: $n=495$; CGGA-mRNA693: $n=420$; CGGA-mRNA325: $n=170$; CGGA mRNA-array_301: $n=158$; GSE16011: $\mathrm{n}=103$ )(Table 1). The GSE35640 dataset contains 65 metastatic melanoma and non-small-cell lung cancer patients who receiving MAGE-A3 antigen-based immunotherapy and was downloaded from the GEO database (https://www.ncbi.nlm.nih.gov/geo/)(Ulloa-Montoya et al. 2013) as an external validation dataset for immunotherapeutic evaluation.

\section{Prognostic Gene Identification and Construction of an Immune Risk Score Model}

We first used univariate Cox regression analysis to examine the prognostic value of the chemokines and chemokine receptors in the TCGA-LGG dataset, and then prognosis-related genes were included for more functional investigation and developed possible risk score using the LASSO Cox regression algorithm. Tuning parameter (lambda) selection was applied in the LASSO model by using 10-fold cross-validation via the minimum and 1-standard error (SE) criteria. Finally, the model with 1-SE criteria was selected to define a four-gene risk score model and their coefficients. Using the following formula, the risk score $=\operatorname{Exp}_{1}{ }^{*} \mathrm{Coe}_{1}+\operatorname{Exp}_{2}{ }^{\star} \mathrm{Coe}_{2}+\ldots . . .+\operatorname{Exp}_{n}{ }^{*} \mathrm{Coe}_{n}$, Exp stands for the mRNA expression value, and Coe indicates the coefficients calculated by LASSO regression analysis. "glmnet", "survival", and "timeROC" R packages were used for the construction of prognostic model.

\section{Protein-Protein Interaction (PPI) Network Construction}

To better understand the interactions between candidate genes, we used the Search Tool for the Retrieval of Interacting Gene/Proteins (STRING) online tool (version 11.0; http://string-bd.org/) (combined score>=0.7 was used as the cut-off criterion) to create a PPI network.

\section{Multi-omics Analysis of Hub Genes}

The differential expression of hub genes between glioma samples and normal brain tissues was evaluated by the GenotypeTissue Expression (GTEX) and TCGA databases, and transcriptomic data were acquired from the UCSC Xena browser (https://xenabrowser.net/datapages/). Protein level exploration of the hub genes in glioma samples and their subcellular localization (immunofluorescence staining) in glioma U251 cell line were performed using the Human Protein Atlas (HPA) database (https://www.proteinatlas.org)(Uhlén et al. 2005; Uhlen et al. 2010). The Oncomine database

(https://www.oncomine.org/resource/login.html) was used to perform a pan-cancer analysis of the differential expression of hub genes, with cutoff values for the probability and fold change of 0.05 and 1.0, respectively. The survival information and the hub gene expression profiles of 32 cancer types in the TCGA dataset, including including acute myeloid leukemia (LAML), adrenocortical carcinoma (ACC), cholangiocarcinoma (CHOL), bladder urothelial carcinoma (BLCA), breast invasive carcinoma (BRCA), cervical squamous cell carcinoma and endocervical adenocarcinoma (CESC), colon adenocarcinoma (COAD), uterine corpus endometrial carcinoma (UCEC), esophageal carcinoma (ESCA), glioblastoma (GBM), head and neck squamous carcinoma (HNSC), kidney chromophobe (KICH), kidney clear cell carcinoma (KIRC), kidney papillary cell carcinoma (KIRP), lymphoid neoplasm diffuse large B-cell lymphoma (DLBC), liver hepatocellular carcinoma (LIHC), lung adenocarcinoma (LUAD), lung squamous cell carcinoma (LUSC), skin cutaneous melanoma (SKCM), mesothelioma (MESO), uveal melanoma (UVM), ovarian serous cystadenocarcinoma (OV), pancreatic adenocarcinoma (PAAD), pheochromocytoma and paraganglioma (PCPG), prostate adenocarcinoma (PRAD), rectum adenocarcinoma (READ), sarcoma (SARC), stomach adenocarcinoma (STAD), testicular germ cell tumor (TGCT), thymoma (THYM), thyroid carcinoma (THCA), and uterine carcinoma (UCS), were obtained from the UCSC Xena browser (https://xenabrowser.net/datapages/). Univariate Cox regression analysis was used to evaluate the prognostic value of the hub genes in 32 cancer types. 


\section{Differentially Expressed Gene (DEG) Identification and Functional Enrichment Analysis}

The DEGs in groups were selected using the $\mathrm{R}$ limma package with a defined standard $|\log \mathrm{FC}|>=1.0$ and an adjusted $\mathrm{P}<0.05$ (Ritchie et al. 2015). Gene Ontology (GO) analysis and gene set enrichment analysis (GSEA) were performed using the OmicShare tool (https://www.omicshare.com), a free online platform for data analysis. Only the enrichment pathways with a $P$ value less than 0.05 and false discovery rate (FDR) q value less than 0.25 were considered significant.

\section{Principal Components Analysis (PCA)}

Immune status in low- and high-risk groups was evaluated by PCA, which was performed by ClustVis (https://biit.cs.ut.ee/clustvis/)(Metsalu and Vilo. 2015), a web tool for visualizing clustering of multivariate data.

\section{Immune Microenvironment Exploration and Immunotherapeutic Efficacy Estimation.}

The gene lists of immune checkpoints and immunoinhibitory molecules were acquired from TISIDB database (http://cis.hku.hk/TISIDB)(Ru et al. 2019). Estimation of Stromal and Immune 『cells in Malignant Tumor tissues using Expression data (ESTIMATE) is a commonly used algorithm that can be applied to calculate immune and stromal scores in $\nabla$ tumor tissues based on expression profiles (Yoshihara et al. 2013). Higher immune scores represent higher immune cells infiltration in cancer microenvironment. Immune scores for the TCGA-LGG dataset were downloaded from the official ESTIMATE website (https://bioinformatics.mdanerson.org/estimate/). $\otimes$ Tracking the Tumor Immunophenotype (TIP) is an online website (http://biocc.hrbmu.edu.cn/TIP/) that provides a user-friendly tool for evaluating anticancer immune activity processes in cancers by using their own gene expression profiles and estimating the abundance of 14 types of immune cells (B cells, CD4 naive, CD4 memory, CD8 naive, CD8+ memory, CD8+ effector, T helper (Th) cells, T cells regulatory (Tregs), monocytes CD16+, monocytes CD14+, dendritic cells (DCs), plasmacytoid DCs (pDCs), natural killer (NK) cells and plasma cells) (Xu et al. 2018). The TIMER2.0 database (https://timer.cistrome.org/) is an online tool for the analysis and visualization of immune cell infiltration among more than 10,000 samples from TCGA cancer types. Six tumor-infiltrating immune subsets, including B cells, CD4+ T cells, CD8+ T cells, macrophages, neutrophils and DCs were investigated (Li et al. 2020). ImmuCellAI (http://bioinfo.life.hust.edu.cn/ImmuCellAI) can be applied to estimate ICB treatment effects on cancers based on gene expression profiles and also to assess the abundance of 24 immune cell types in cancers (Miao et al. 2020). CIBERSORT provides an estimation of the abundances of 22 immune cell types (naive B cells, memory B cells, plasma cells, CD8 T cells, naive CD4 T cells, resting \memory CD4 T cells, activated memory CD4 T cells, follicular helper T (Tfh) cells, Tregs, gamma delta T (Tgd) cells, resting NK cells, activated NK cells, monocytes, macrophages M0, macrophages M1, macrophages M2, resting DCs, activated DCs, resting mast $\$ cells, activated mast cells, eosinophils and neutrophils) and has been widely used for evaluating the immune cells infiltration levels in cancers (Newman et al. 2015). A previous study comprehensively analyzed the immune subtypes of the TCGA cancer types based on five immune expression signatures, which divided 30 non-hematologic cancer types in 6 immune subtypes, namely C1 (wound healing), C2 (IFN-ydominant), C3 (inflammatory), C4 (lymphocyte depleted), C5(immunologically quiet) and C6 (TGF-ßBdominant)(Thorsson et al. 2018). The TCGA Pan-Cancer cohort contained cancer immune subtypes were acquired from the UCSC Xena browser (https://xenabrowser.net/datapages/). TIDE (Jiang et al. 2018), a computational algorithm to model two primary mechanisms of tumor immune evasion, the induction of T cell dysfunction in tumors with high infiltration of cytotoxic $T$ lymphocytes (CTLS) and the prevention of T cell infiltration in tumors with low CTL levels, were used to evaluate the ICB treatment effects on LGG patients. A gene list of 66 immune-gene markers was acquired from a previous study, which was manually curated to represent the broad landscape of immune profile (Cancer Genome Atlas Research Network 2017). Another gene markers of 16 types of immune cells were obtained from our previous study to investigate the relationship between immune cells infiltration levels and a risk score model (Qi et al. 2021).

\section{Statistical Analysis}

All graphic and statistical work was completed using GraphPad Prism software (version no. 7; GraphPad Software, Inc.), R language (3.6.3), SPSS software (version 21.0; IBM Corp.) and the online tools mentioned above. Kaplan-Meier survival 
analysis was performed to compare OS between two cohorts using the log-rank test. We used unpaired t-tests to compare 2 groups and ANOVA to compare three groups. The median value in each step was used to define the high (>=median value) and low (<median value) groups. Correlation analysis was performed by Pearson's correlation coefficient analysis. A receiver operating characteristic (ROC) curve was utilized to assess the sensitivity and specificity, and the area under the curve (AUC) was used to present the efficacy. A $p<0.05$ was considered to indicate a statistically significant difference in all steps.

\section{Results}

\section{Candidate Genes Identification and Risk Score Model Construction}

By using the TISIDB database (Ru et al. 2019), after removing data with the exclusive criteria mentioned above, we finally acquired gene expression files of 46 genes and 495 LGG patients from the TCGA-LGG dataset (Fig. 1A). Univariate Cox regression analyses were used to investigate the prognostic value of these genes in LGG patients based on the TCGA-LGG dataset and 24 prognosis-related genes were identified (Fig. 1B). A heatmap was used to show the correlation between 24 prognostic genes, most of which showed a significant correlation with others (Fig. 1C). To better understand the interactions among these prognostic genes, we conducted a PPI network analysis, which consisted of 24 nodes and 144 edges (Fig. 1D).

The LASSO regression predictive formula (lambda.1-SE criteria) was used to identify the accurate prognosis-related genes and their coefficients (Fig. 1E-F). The lambda value was 0.0776 . The formula built by LASSO regression was as follows: risk score $=0.145{ }^{*} \mathrm{CCR} 4-0.040{ }^{*} \mathrm{CX} 3 \mathrm{CL} 1+0.055{ }^{*} \mathrm{CXCL} 10+0.042{ }^{*} \mathrm{CXCL} 11$. To confirm the prognostic value of the four genes in LGG patients, we used Kaplan-Meier curves to explore the prognostic value of the four genes in the TCGA-LGG, CGGA mRNA-325 and the CGGA mRNA-693 datasets, and the results supported their valuable prognostic effects on LGG patients (Fig. S1A-L).

\section{Multi-omics Analyses of the Genes in the Risk Score Model}

We first used the GTEX and TCGA databases to compare the differential expression of the four genes between glioma samples and normal brain tissues. The results revealed higher expression of CCR4, CX3CL1, CXCL10 and CXCL11 was found in glioma samples compared with normal brain tissues (Fig. 2A-D). Protein level exploration of the four genes was performed by immunohistochemistry (IHC) staining from the HPA database (Uhlén et al. 2005; Uhlen et al. 2010). We observed a weak intensity of CCR4 in glioma samples. CX3CL1 was found to be strong and moderate intense in glioma samples. Moderate and weak intensity staining of CXCL11 was found in glioma tissues, but the CXCL10 data were not acquired from this database (Fig. 2E).

In view of the important roles of the four genes in LGG patients, we further investigated the expression of these genes in multiple cancers using the Oncomine database. High expression of CCR4 was found in brain and CNS cancer, breast cancer, head and neck cancer, leukemia, lung cancer, lymphoma and melanoma (Fig. 2F). High expression of CX3CL1 was found in brain and CNS cancer, breast cancer, cervical cancer, colorectal cancer, gastric cancer, head and neck cancer, kidney cancer, leukemia, liver cancer, lymphoma, ovarian cancer, pancreatic cancer, and sarcoma; however, lower expression of CX3CL1 was found in more of the above cancer datasets compared with corresponding normal tissues (Fig. 2F). Elevated expression of CXCL10 was found in cancer samples compared with normal tissues, including bladder cancer, brain and CNS cancer, breast cancer, cervical cancer, colorectal cancer, esophageal cancer, gastric cancer, head and neck cancer, kidney cancer, leukemia, liver cancer, lung cancer, lymphoma, melanoma, ovarian cancer, pancreatic cancer, prostate cancer and sarcoma (Fig. 2F). High expression of CXCL11 was found in bladder cancer, brain and CNS cancer, breast cancer, cervical cancer, colorectal cancer, esophageal cancer, gastric cancer, head and neck cancer, kidney cancer, leukemia, liver cancer, lymphoma, ovarian cancer, prostate cancer and sarcoma (Fig. 2F). To characterize the intracellular localization of these four genes in glioma cell lines, we assessed the distribution within the microtubules and nucleus in glioma U251 cell line. Immunofluorescence results showed that CCR4 and CX3CL1 were mainly localized in plasma membrane (CXCL10 and CXCL11 were not acquired from the HPA database, Fig. 2G). Next, we used univariate Cox regression analyses to explore the prognostic value of the four genes in 32 cancer types (LGG was excluded) in the TCGA dataset (Fig. 2H). CCR4 showed 
prognostic value in UVM, SKCM, LUAD, KIRC, HNSC and BRCA. CX3CL1 showed prognostic association with KIRP, KIRC and CESC. The prognostic value of CXCL10 was determined in UVM, SKCM, ESCA, LAML, BRCA, THYM and PAAD. For CXCL11, the prognostic value was found in UVM, SKCM, BRCA and PAAD.

All the results revealed the prognostic value of CCR4, CX3CL1, CXCL10 and CXCL11 in multiple human cancers and supported their further study. These results undoubtedly demonstrated that the expression of four genes was heterogeneous in multiple cancers, and their potential value deserves further exploration.

\section{High-Risk Scores Predict Malignant Phenotypes of LGG Patients}

To elucidate the function of our risk score model in malignant phenotypes of LGG patients, we used the TCGA-LGG, CGGA mRNA-325 and CGGA mRNA-693 CGGA mRNA array_301 datasets to compare risk scores with the WHO grades, IDH-1 status, age, sex and 1p19q codeletion status. The results in all the datasets showed consistency in evaluation of risk scores in terms of WHO grade, IDH-1 status and 1p19q codeletion status. WHO grade III, IDH-1 wild type and 1p19q non-codeleted LGG patients had higher risk scores than their corresponding counterparts (Fig. 3). However, for different ages and sexes, the results were not consistent among the four datasets. These results indicated that our risk score model could effectively predict malignant phenotypes of LGG patients.

\section{The Prognostic Value of Risk Score Model in LGG Patients}

To determine whether the four-gene risk score model had reliable prognostic value in LGG patients. We defined the TCGALGG dataset as an internal training dataset, of which all LGG patients were subdivided into high- and low-risk score groups based on the median risk scores. LGG patients with high-risk scores had worse prognoses (Fig. 4A). For WHO grade II and III glioma patients, high-risk scores predicted a shorter OS. We used ROC curves to assess the accuracy of our risk score model in 1-, 3- and 5-year OS for LGG patients, which showed that the AUC was 0.849, 0.704, and 0.641, respectively (Fig. 4A). Meanwhile, we used four independent datasets from the CGGA (mRNA-325, mRNA-693, and mRNA array_301) and GEO (GSE16011) databases as external validation sets to estimate the prognostic value of the risk score model in LGG patients. In the CGGA mRNA-325 dataset, all LGG patients and WHO grade III LGG patients with higher risk scores had shorter OS; the AUC value of the ROC curve for 1-, 3- and 5-year OS were $0.769,0.740$, and 0.743 , respectively (Fig. 4B). Consistent results were also found in the CGGA mRNA-693, mRNA array_301 and GSE16011 datasets (Fig. 4C-E). Multivariate Cox regression analysis was then performed in the TCGA-LGG dataset to investigate the independent role of the risk score model in the OS of patients with LGG. The results indicated that after adjusting for age, sex, 1p19q codeletion status, and IDH- 1 status, the risk score still affected the OS of patients with LGG (Table S1).

Consistently, our results demonstrate that the novel four-gene risk signature was an effective prognostic biomarker meriting further study in clinical application.

\section{Prognostic Evaluation of Risk Score Model in Pan-Cancer Datasets}

Through the above study, we found that our risk score model effectively predicted the OS of LGG patients. To clarify its function in pan-cancer prognosis, we analyzed our risk model in 32 cancer types from the TCGA database (LAML, ACC, CHOL, BLCA, BRCA, CESC, COAD, UCEC, ESCA, GBM, HNSC, KICH, KIRC, KIRP, DLBC, LIHC, LUAD, LUSC, SKCM, MESO, UVM, OV, PAAD, PCPG, PRAD, READ, SARC, STAD, TGCT, THYM, THCA and USC) using Kaplan-Meier curves, and ROC curves were used to evaluate the accuracy of our model in OS. The results indicated that our risk score model showed prognostic value in BRCA, HNSC, SKCM and UVM (Fig. 5), but no significant difference was found in other types of cancers (Fig. S2). However, the effects of the risk score on these four cancers showed difference; BRCA, SKCM and HNSC patients who had high-risk scores presented longer OS, but UVM patients with high-risk scores had a shorter OS. We further used multivariate Cox regression analyses to investigate the prognostic value of the risk score model in BRCA, SKCM, UVM and HNSC. The results confirmed that the present risk score model displayed independent roles in the prognosis of the four cancers (Table S2-5). The results indicated that the four-gene risk score model had distinct prognostic effects on these cancers. 


\section{The Present Risk Score Model Indicated Distinct Immune Subtypes of the TCGA Cancers}

In the above study, we revealed the prognostic value of the present risk score model in the TCGA-LGG, BRCA, SKCM, UVM and HNSC datasets, however, the relationship between risk scores and OS were not consistent in theses five cancers. We further compared the risk scores with main immune subtypes of BRCA, HNSC, LGG, UVM and SKCM patients (Fig. 6A and Table S6). The results showed that the risk score distribution in immune subtypes of these five cancers were different (Fig. 6B-F), the LGG and UVM patients in C3 type (inflammatory) had higher risk scores (Fig. 6D,F), and the patients with BRCA, SKCM and HNSC, who were in the C2 type (IFN-gamma dominant) had higher risk scores (Fig. 6B,C,E). The composition of immune subtypes in these five cancers between the low- and high-risk score groups is shown in Fig. 6G-K. In summary, the present risk score model reflected distinct immune subtypes of these five cancers and, therefore, potentially explained its distinct prognostic value in these cancers.

\section{Low- and High-Risk Groups Displayed Distinct Immune Status}

To further investigate the associated signaling pathways that were activated in the high-risk score group, we performed GSEA comparing the high- and low-risk score groups. Gene sets from the TCGA-LGG dataset that were enriched in the highrisk score group were related to the processes involved in tumor proliferation and immune response (Fig. 7A), such as HALLMARK_INTERFERON_GAMMA_RESPONSE, HALLMARK_INFLAMMATORY_RESPONSE,HALLMARK_INTERFERON_ALPHA_RESPNSE,HALLMARK_TNFA_SIGNALING_VIA_ NFKB,HALLMARK_IL6_JAK_STAT3_SIGNALING,HALLMARK_EPITHELIAL_MESENCHYMAL_TRANSITION,HALLMARK_ ANGIGENESIS,HALLMARK_IL2_STAT5_SIGNALING,HALLMARK_COMPLEMENT and HALLMARK_ALLOGRAFT_REJECTION. Consistent results were also found in KEGG and GO enrichment analyses, indicating that the high-risk score group might be associated with cancer-related pathway activation and immune response dysfunction (Fig. 7B and Fig. S3A-C).

We further identified the DEGs between high- and low-risk score groups, and 754 DEGs were selected, including 610 upregulated and 144 down-regulated genes (Fig. S3D). The top $10 \mathrm{GO}$ terms ranked by negative log10 $\mathrm{P}$ value were listed (Fig. $7 C, D)$. For biological process (BP), the DEGs were mainly enriched in immune response, immune system process, response to external stimulus, regulation of immune system process, and cell activation. For cellular component (CC), the DEGs were enriched in extracellular matrix, cell surface, plasma membrane part, collagen-containing extracellular matrix and extracellular region. The DEGs in molecular function (MF) were mainly enriched in extracellular matrix structural constituent, signaling receptor activity, signaling receptor binding, molecular transducer activity and transmembrane signaling receptor activity.

To further investigate the immune microenvironment in LGG patients, we selected 66 immune gene markers to reflect the broad landscape of the immune profile, which has been used in previous studies (Bidkhori et al. 2018; Zhou et al. 2021). The genes expression profile between the low- and high-risk score groups is shown in a heatmap (Fig. S3E). We then conducted PCA to investigate the difference between low- and high-risk groups based on a 66 immune-related gene signature of the TCGA-LGG dataset. The results showed that the low- and high-risk groups were generally distributed in different directions (Fig. 7E). Thus, the risk score system could reflect the different immune statuses of LGG patients.

\section{The Different Anticancer Immune Activities Between High- and Low-Risk Groups}

The anticancer immune activity processes can be conceptualized as a series of stepwise events in the cancer-immunity cycle (Xu et al. 2018), as follows: release of cancer cell antigens (step 1); presentation of cancer antigens (step 2); priming and activation (step 3); trafficking of immune cells to tumors (step 4); infiltration of immune cells into tumors (step 5); recognition of cancer cells by T cells (step 6); and killing of cancer cells (step 7). Through the TIP online tool (Xu, et al, 2018), we estimated the different anticancer immune scores between the high- and low-risk score groups. We found higher anticancer immune scores in the high-risk score groups in step1 (release of cancer cell antigens), step4 (T cell recruiting, CD4 T cell recruiting, CD8 T cell recruiting, Th1 cell recruiting, dendritic cell recruiting, Th22 cell recruiting, macrophage recruiting, monocyte recruiting, neutrophil recruiting, NK cell recruiting, eosinophil recruiting, basophil recruiting) 
and step 5 (infiltration of immune cells into tumors). Conversely, lower anticancer immune scores in the high-risk score groups were found in step 3 (priming and activation), step 4 (B cell recruiting) and step 7 (killing of cancer cells) (Fig. 8A).

Next, we conducted PCA to investigate the different anticancer immune activity scores between low- and high-risk groups based on the TCGA-LGG dataset. The results showed that anticancer activity scores were generally distributed in different directions (Fig. 8B). Thus, our results demonstrated that the patients in the high-risk and low-risk groups have different anticancer immune processes.

\section{High-Risk Scores Indicated Immune Microenvironment Alterations in LGG Patients}

As reported in previous studies, the levels of immune cell infiltration could predict the prognosis of patients with glioma (Gieryng et al. 2017; Yin et al. 2020). We used three algorithms (TIMER, TIP and CIBERSORT) to calculate the immune cell infiltration levels in the TCGA-LGG dataset (Li et al. 2020; Newman et al, 2015; Xu et al. 2018). We demonstrated that LGG patients with high-risk scores have higher immune cells infiltration levels of CD4+ T cells, CD8+ T cells, B cells, neutrophils, DCs and macrophages in the TIMER database (Fig. 9A). For the TIP algorithm, significant differences between high- and lowrisk groups were found in B cells, naive CD 4 cells, naive CD8 cells, effector CD8 cells, Th cells, CD14 monocytes, dendritic cells, DCs, pDCs, NK cells and plasma (Fig. 9B). Twenty-two types of immune cell abundance were explored in the CIBERSORT method, of which significant differences between high- and low-risk groups were found in naive B cells, B cell plasma, CD8+ T cells, naive CD4+ T cells, memory resting CD4+ T cells, Tfh, activated NK cells, monocyte, M1 macrophages, M2 macrophages, resting mast cells and neutrophils (Fig. 9C).

Next, the correlation between our risk score and fifty-seven gene markers (Table S7) was performed by Pearson correlation coefficient analysis. Our risk scores were positively correlated (Pearson's $r>=0.3, P<0.05$ ) with the expression of most gene markers. Forty-one, thirty and thirty-seven gene markers were positively correlated with risk scores in the TCGA-LGG, CGGAmRNA693 and CGGA mRNA-325 datasets (45 gene markers were found in the CGGA dataset), respectively (Fig. 9D-F). Based on median risk scores, the results from PCA showed that the immune cell gene markers expression was divided into different directions (Fig. 9G-I).

Overall, our risk score model can reflect different immune cell infiltration levels in LGG patients.

\section{LGG Patients with High Risk Scores Present Treatment Resistance}

Immune checkpoints and immunoinhibitory molecules play important roles in cancer immune suppression, and their inhibitors are promising strategies for cancer treatment (Rizvi et al. 2015). Next, we investigated the relationship between the risk score and the expression of critical immune checkpoints and immunoinhibitory molecules in the TCGA-LGG dataset. We found that the LGG patients with high-risk scores showed significantly elevated expression of PDCD1, CD274, CTLA-4, LAG-3 and other molecules (Fig. 10A). Through the ESTIMATE algorithm (Yoshihara et al. 2013), we estimated the relationship between our risk scores and immune, stromal and ESTIMATE scores, and found that patients in the high-risk score group have higher immune, stromal and ESTIMATE scores than those in the low-risk score group (Fig. 10B-D). Using TIDE and ImmuCellAI methods (Jiang et al. 2018; Miao et al. 2020), we predicted the response of LGG patients to ICB treatment in the TCGA-LGG dataset. We found that LGG patients in the "non-response" group had higher risk scores and that low-risk score groups had a higher rate of "response" to ICB treatment (Fig. 10E,F). We used GSE35640 dataset to validate the effect of our risk score model on melanoma and non-small-cell lung cancer patients who received ICB treatment. The risk scores in the "response" and "non-response" groups were significantly different, and the patients with low-risk scores had a higher rate of "non-response" to ICB treatment (Fig. 10G).

Based on the median risk scores, the LGG patients with high-risk scores in the "non-response" groups had a shorter OS, but no significance was found in the "response" groups (Fig. 10H-I). All the results showed that our risk score model was not only associated with high levels of immune cell infiltration but might also become a valuable indicator for immunotherapy in the future. 


\section{Discussion}

Glioma is a deadly disease severely threatens patients' health because existing treatment strategies remain uncurable (Stupp et al. 2005). The highly invasive feature and rapid recurrence after standard treatments create the glioma prognosis dilemma. The identification of key molecules in gliomas has provided superior diagnostic and prognostic value, and as a result, our understanding of glioma behavior has rapidly evolved (Louis et al. 2016). However, as a type of heterogeneous tumor, the dismal prognosis and rapid recurrence after surgery prompted us to search for more effective molecular biomarkers to overcome the limited roles of a single molecule in glioma diagnosis and prognosis.

The important roles of chemokines and chemokine receptors in cancers have been extensively reported. Mounting evidence suggests that chemokines and chemokine receptors are potential therapeutic targets for a variety of diseases (Mollica et al. 2019; Nagarsheth et al. 2017). In the present study, we comprehensively analyzed the prognostic value of chemokines and their receptors in LGG patients, and a four-gene risk score model was created. Then we analyzed the differential expression and prognostic value of the four genes in multiple cancers. The results confirmed the heterogeneous effects of the four genes on prognostic estimation and expression profiles among the pan-cancer analysis. The clinical functions of the four genes in cancers have also been detected in previous studies. Many studies have highlighted the important roles of CCR4 in cancer immune microenvironment and potential target in immunotherapy (Berlato et al. 2017). In the context of CCR4 expression, CCL17 and CCL22 can promote the stemness of cancer cells, drug resistance, proliferation of cancer cells and therefore result in cancer cell migration and epithelial mesenchymal transition (EMT)(Korbecki et al. 2020). CCR4 expression has also been linked to lymph node metastasis and omental milky spots metastasis and is overexpressed in many hematological malignancies, such as adult T-cell leukemia (ATL) and cutaneous T-cell lymphoma (CTL)(Korbecki et al. 2020; Mollica et al. 2019). The human anti-CCR4 antibody mogamulizumab eliminates tumor cells via antibody-dependent cellular cytotoxicity (ADCC) and has been applied to the treatment of relapsed/refractory ATL (Fuji et al. 2018). CCR4 is also considered a promising target for solid tumors because of its activity in modulating leukocyte infiltration and small molecule antagonists of CCR4 with less harmful side effect are in development and considerably improve the efficacy of cancer vaccines in different preclinical tumor models by preventing Tregs induction, such as melanoma, lung, and colon cancers (Beziaud et al. 2018; Mollica et al. 2019). CX3CL1 is constitutively expressed in the central nervous system by neurons and astrocytes, controlling neuronal survival and neurotransmission, and it has been shown to mediate both NK cell-dependent and T cell-dependent antitumor activity (Groblewska et al. 2020). In the tumor niche, the CX3CL1-CX3CR1 axis plays important anticancer roles, as it allows for the migration of immature dendritic cells to the cancer cells based on CX3CL1 expression. An increased expression of CX3CL1 in a tumor is correlated with the accumulation of anticancer CD8+ T cells, dendritic cells and NK cells in the tumor (Park et al. 2012). By Ren's study, significantly higher CX3CL1 mRNA levels were in IDH-1 mutation type glioma patients and presented longer OS (Ren et al. 2019). Our results were consistent with their conclusions, and higher levels of CX3CL1 showed protective effects on LGG patients. However, other studies from different groups have reached opposite conclusions. Erreni and colleagues found that the levels of CX3CL1 significantly correlated with glioma malignancy and inversely correlated with OS (Erreni et al. 2010). These disparate findings need in-depth investigation to address the underlying mechanisms. CXCL10 can interact with CXCR3 to exhibit dual functions in tumorigenesis depending on the spliced variant: CXCR3-A promotes cell proliferation, and CXCR3-B exerts growth inhibition. In breast cancers, CXCL10 could contribute to tamoxifen resistance and promote breast cancer proliferation via estrogendependent and estrogen-independent pathways (Wu et al. 2020). Glioma cells showed increased production of CXCL10, which induced DNA synthesis in grade III astrocytoma and grade IV GBM cells and promoted ERK1/2 activation, leading to cancer cells proliferation (Maru et al. 2008). However, protective effects have also been identified in Chen's study, where low CXCL10 expression was correlated with unfavorable OS in colorectal cancer patients (Chen et al. 2020). CXCL11 is induced by IFN-gamma and IFN-beta, and secreted by epithelial cells, neutrophils, endothelial cells, eosinophils, monocytes, stromal cells and cancer cells. The affinity of CXCL11 for CCR3 is highest of the three selective ligands (CXCL9/10/11)(Tokunaga et al. 2018); alternately, CXCL11 can bind to CXCR7, which is associated with invasiveness and reduces tumor cell apoptosis. Its high expression in cancers has been reported and correlates with a poor prognosis in many cancer types. In Gao's study, down-regulation of CXCL11 inhibits colorectal cancer cell growth and EMT (Gao et al. 2018). However, an enhanced

Page 10/27 
anticancer effect was identified by Liu and colleagues, who created a tumor-selective oncolytic vaccinia virus with CXCL11 (vvDD-CXCL11) model (Liu et al. 2016), which induced a systematic increase in tumor-specific IFN gamma-producing CD8+ T cells in the spleen and other lymph organs, indicating the induction of systemic antitumor immunity. This effect was associated with enhanced therapeutic efficacy and a survival benefit in tumor-bearing mice treated with vVDD-CXCL11, mediated by CD8+ T cells and IFN gamma but not CD4+ T cells. Therefore, the underlying mechanisms remain to be elucidated in the future.

Although the molecular functions of the four genes have been mentioned in previous studies, an immune risk score model consisting of the above four genes in glioma has not been reported. A risk score system based on candidate genes has been widely used to predict cancer prognosis and conventional clinical parameters, such as lymph node metastasis in colorectal cancer, pathological classification in glioma, and chemotherapy resistance in breast cancers, and it also reflects immune cell infiltration or immunotherapy resistance in cancers (Farmer et al. 2009; Li et al. 2020b; Lin et al. 2020). In the present study, LGG patients who have higher risk scores (more than median risk scores) exhibited more conventional malignant phenotypes, such as higher WHO classifications, the IDH-1 wild type or the 1p19q non-codeletion type.

Furthermore, previous data showed that many risk score models play predictive roles in the OS of glioma patients (Lin et al. 2020; Yin et al. 2020). We fully validated the four-gene risk score model in 5 independent datasets, which presented consistent results. However, whether the risk score model could reflect other cancers prognosis has not been investigated. We chose 32 type cancers from the TCGA database, applied our risk score model to these cancers and finally identified the prognostic value in 4 cancers (BRCA, SKCM, UVM and HNSC). However, the prognostic performance in four cancers was different, among which the high-risk scores in UVM predicted a shorter OS; conversely, in HNSC, SKCM and BRCA predicted a longer OS. As different cancer immune subtypes may present distinct prognoses, we further analyzed the relationship between risk scores and immune subtypes in these five cancers (LGG, BRCA, HNSC, UVM and SKCM) by Thorsson's classification (Thorsson et al. 2018), which showed that the risk scores distribution in LGG and UVM had similar performance: cases in the C3 immune type had higher risk scores, but for BRCA, HNSC and SKCM, cases in the C2 immune type had higher risk scores. The different risk score distributions among these cancer immune subtypes might lead to their variable functions in prognostic prediction for these cancers.

In the setting of different risk scores (high vs. low), we found that immune gene expression profiles in the TCGA-LGG dataset were divided into two sections by PCA, indicating that the immune status of LGG patients in the low-risk group was quite different from those in the high-risk group. By applying three commonly used algorithms (TIMER, TIP and CIBERSORT) (Li et al. 2020; Newman et al. 2015; Xu et al. 2018), we found that the infiltrating levels of a large number of immune cells between the high- and low-risk score groups were significantly different. This finding confirmed and further supported our conclusion that the risk score model was an effective method to evaluate immune cell infiltration in LGG patients. Considering its effects on immune cells infiltration estimation, we speculated that the anticancer immune process between these two groups was distinct. The anticancer immune response can be conceptualized as a series of stepwise events in the cancerimmunity cycle (Xu et al. 2018). The TIP tool estimates the relative activity of different stages of the immune response from the release of cancer cell antigens to the killing of cancer cells. In each step, the scores represent the activities of the anticancer processes (Xu et al. 2018). A significant difference of anticancer immune scores between the high- and low-risk score groups determined in most anticancer steps, which revealed that the risk score model potently reflects the anticancer processes in LGG patients.

Immune checkpoint blockades (ICB) therapy has emerged as a promising treatment option for advanced cancers and has revolutionized the treatment landscape and improved the prognoses for many malignancies (Hodi et al. 2010; Rizvi et al. 2015). However, glioma has its peculiar features in immune response, including an immune-privileged site, scarce infiltrating lymphocytes and the presence of a large number of immunosuppressive cells, indicating poor response to ICB treatment. However, a comparable evaluation of the risk score model of the chemokines and their receptors has not been performed in glioma and the roles of their risk score model play in ICB treatment remain unknown. TIDE is a computational algorithm that is used to model two primary mechanisms of tumor immune evasion and has been extensively used to predict ICB effects in

Page $11 / 27$ 
cancers, providing a convenient method to evaluate the ICB treatment based on gene expression profiles (Jiang et al. 2018). Here, we compared our risk score model with the key immune checkpoints expression in the TCGA-LGG dataset and the results showed that high-risk score groups accompanied with higher immune checkpoints expression. The effects of ICB on LGG patients were fully evaluated by TIDE method and LGG patients showed "non-response" to ICB treatment have higher risk scores, meanwhile, the results were confirmed by ImmuCellAl algorithm (Miao et al. 2020).

ICB treatment in melanoma has achieved promising results (Hodi et al. 2010), in the present study, we identified the different prognostic value of our risk score model in melanoma patients compared with in LGG patients, and the relationship between ICB treatment effects and the present risk scores in these two cancers was quite different. Such a difference may derive from the distinct biological processes and special immune features of these two cancers. However, the number of the clinical samples is limited in this study, more prospective studies are needed in the future. In summary, our risk score model is a valuable model for indicating LGG immune infiltration and immune therapeutic effects.

Although the results from this study were inspiring, limitations are still present. These results were mainly derived from retrospective studies with distinct sequencing platforms and ethnic groups, which could inevitably introduce bias during the data analyses. The protein levels validation was not adequate. Therefore, prospective studies and clinical samples validation should be performed in the future to overcome these difficulties. In vivo and in vitro studies are urgently needed to investigate the biological function of the hub genes and their anticancer immune processes in LGG. In addition, the controversy of the molecular functions for CCR4, CX3CL1, CXCL10 and CXCL11 has not been resolved. These unresolved challenges will require in-depth validation of the underlying mechanisms for the selected genes and their risk score system in the future, which will unquestionably constitute the focus of our next step research.

Overall, these results suggest that our risk score model is a valuable model that not only indicates conventional clinical parameters of LGG patients but also reflects immune cell infiltration levels and preliminarily elucidates its role in immunotherapy for LGG patients.

\section{Conclusion}

In summary, we comprehensively analyzed the prognostic role of chemokines and chemokine receptors in LGG patients, and identified a four-gene signature that could be used to create a novel risk score model. The prognosis and immune microenvironment between the low- and high-risk score groups were quite different, suggesting that they are potential indicators for immunotherapy in LGG patients.

\section{Declarations}

Author Contribution SW O was involved in the conceptualization of the study and in funding acquisition. CX Q and SW O were responsible for conception and design of the study. CX Q, LL and NN L made the data collection, statistical analyses, and writing of the manuscript. All authors contributed to data analysis, drafting and revising the article, gave final approval of the version to be published, and agreed to be accountable for all aspects of the work.

Funding This study was funded by the Liaoning Province Special Professor Project (Grant no. 3110517003).

Availability of Data and Material The datasets for this study can be found in the UCSC Xena browser (TCGA-LGG dataset, https://xenabrowser.net/datapages/), GEO (GSE16011, https://www.ncbi.nlm.nih.gov/gds/) and CGGA (CGGA-mRNA693, CGGA-mRNA325 and CGGA-mRNA301, http://www.cgga.org.cn/).

\section{Declarations}

Ethics Approval Not applicable. 
Consent for Publication All authors agree to publish the manuscript.

Consent to Participate Not applicable.

Competing Interests The authors declare no competing interests.

\section{References}

1. Balkwill F (2004) Cancer and the chemokine network. Nat Rev Cancer 4(7):540-550. https//doi.org/10.1038/nrc1388

2. Bao ZS, Chen HM, Yang MY, Zhang CB, Yu K, Ye WL, Hu BQ, Yan W, Zhang W, Akers J, Ramakrishnan V, Li J, Carter B, Liu YW, Hu HM, Wang Z, Li MY, Yao K, Qiu XG, Kang CS, You YP, Fan XL, Song WS, Li RQ, Su XD, Chen CC, Jiang T (2014) RNA-seq of 272 gliomas revealed a novel, recurrent PTPRZ1-MET fusion transcript in secondary glioblastomas. Genome Res 24(11):1765-1773. https//doi.org/10.1101/gr.165126.113

3. Ben-Baruch A (2008) Organ selectivity in metastasis: regulation by chemokines and their receptors. Clin Exp Metastasis 25(4):345-356. https//doi.org/10.1007/s10585-007-9097-3

4. Berlato C, Khan MN, Schioppa T, Thompson R, Maniati E, Montfort A, Jangani M, Canosa M, Kulbe H, Hagemann UB, Duncan AR, Fletcher L, Wilkinson RW, Powles T, Quezada SA, Balkwill FR (2017) A CCR4 antagonist reverses the tumorpromoting microenvironment of renal cancer. J Clin Invest 127(3):801-813. https//doi.org/10.1172/jci82976

5. Beziaud L, Boullerot L, Tran T, Mansi L, Marie-Joseph EL, Ravel P, Johannes L, Bayry J, Tartour E, Adotévi O (2018) Rapalog combined with CCR4 antagonist improves anticancer vaccines efficacy. Int J Cancer 143(11):3008-3018. https//doi.org/10.1002/ijc.31842

6. Bidkhori G, Benfeitas R, Klevstig M, Zhang C, Nielsen J, Uhlen M, Boren J, Mardinoglu A (2018) Metabolic network-based stratification of hepatocellular carcinoma reveals three distinct tumor subtypes. Proc Natl Acad Sci U S A 115(50):E11874-e11883. https//doi.org/10.1073/pnas.1807305115

7. Brat DJ, Verhaak RG, Aldape KD et al (2015) Comprehensive, integrative genomic analysis of diffuse lower-grade gliomas. N Engl J Med 372(26):2481-2498. https//doi.org/10.1056/NEJMoa1402121

8. Cancer Genome Atlas Research Network (2017) Comprehensive and integrative genomic characterization of hepatocellular carcinoma. Cell 169(7):1327-1341. .e23. https//doi.org/10.1016/j.cell.2017.05.046

9. Chen J, Chen QL, Wang WH, Chen XL, Hu XQ, Liang ZQ, Cao YB, Cao YM, Su SB (2020) Prognostic and predictive values of CXCL10 in colorectal cancer. Clin Transl Oncol 22(9):1548-1564. https//doi.org/10.1007/s12094-020-02299-6

10. Cheng F, Zhang P, Xiao Q, Li Y, Dong M, Wang H, Kuang D, He Y, Duan Q, Mao F, Wang B, Guo D (2019) The prognostic and therapeutic potential of LRIG3 and soluble LRIG3 in glioblastoma. Front Oncol 9:447.

https//doi.org/10.3389/fonc.2019.00447

11. Erreni M, Solinas G, Brescia P, Osti D, Zunino F, Colombo P, Destro A, Roncalli M, Mantovani A, Draghi R, Levi D, Rodriguez YBR, Gaetani P, Pelicci G, Allavena P (2010) Human glioblastoma tumours and neural cancer stem cells express the chemokine CX3CL1 and its receptor CX3CR1. Eur J Cancer 46(18):3383-3392.

https//doi.org/10.1016/j.ejca.2010.07.022

12. Fang S, Liang J, Qian T, Wang Y, Liu X, Fan X, Li S, Wang Y, Jiang T (2017) Anatomic location of tumor predicts the accuracy of motor function localization in diffuse lower-grade gliomas involving the hand knob area. AJNR Am J Neuroradiol 38(10):1990-1997. https//doi.org/10.3174/ajnr.A5342

13. Farmer P, Bonnefoi $H$, Anderle $P$, Cameron D, Wirapati P, Becette V, André S, Piccart M, Campone M, Brain E, Macgrogan G, Petit T, Jassem J, Bibeau F, Blot E, Bogaerts J, Aguet M, Bergh J, Iggo R, Delorenzi M (2009) A stroma-related gene signature predicts resistance to neoadjuvant chemotherapy in breast cancer. Nat Med 15(1):68-74.

https//doi.org/10.1038/nm.1908

14. Fuji S, Utsunomiya A, Inoue Y, Miyagi T, Owatari S, Sawayama Y, Moriuchi Y, Choi I, Shindo T, Yoshida SI, Yamasaki S, Yamaguchi T, Fukuda T (2018) Outcomes of patients with relapsed aggressive adult T-cell leukemia-lymphoma: clinical 
effectiveness of anti-CCR4 antibody and allogeneic hematopoietic stem cell transplantation. Haematologica 103(5):e211-e214. https//doi.org/10.3324/haematol.2017.184564

15. Gao YJ, Liu L, Li S, Yuan GF, Li L, Zhu HY, Cao GY (2018) Down-regulation of CXCL11 inhibits colorectal cancer cell growth and epithelial-mesenchymal transition. Onco Targets Ther 117333-117343.

https//doi.org/10.2147/ott.s167872

16. Gieryng A, Pszczolkowska D, Walentynowicz KA, Rajan WD, Kaminska B (2017) Immune microenvironment of gliomas. Lab Invest 97(5):498-518. https//doi.org/10.1038/labinvest.2017.19

17. Gravendeel LA, Kouwenhoven MC, Gevaert O, de Rooi JJ, Stubbs AP, Duijm JE, Daemen A, Bleeker FE, Bralten LB, Kloosterhof NK, De Moor B, Eilers PH, van der Spek PJ, Kros JM, Sillevis Smitt PA, van den Bent MJ, French PJ (2009) Intrinsic gene expression profiles of gliomas are a better predictor of survival than histology. Cancer Res 69(23):90659072. https//doi.org/10.1158/0008-5472.can-09-2307

18. Gravina GL, Mancini A, Marampon F, Colapietro A, Delle Monache S, Sferra R, Vitale F, Richardson PJ, Patient L, Burbidge S, Festuccia C (2017) The brain-penetrating CXCR4 antagonist, PRX177561, increases the antitumor effects of bevacizumab and sunitinib in preclinical models of human glioblastoma. J Hematol Oncol 10(1):5. https//doi.org/10.1186/s13045-016-0377-8

19. Groblewska M, Litman-Zawadzka A, Mroczko B (2020) The role of selected chemokines and their receptors in the development of gliomas. Int J Mol Sci 21(10). :https//doi.org/10.3390/ijms21103704

20. Hodi FS, O'Day SJ, McDermott DF, Weber RW, Sosman JA, Haanen JB, Gonzalez R, Robert C, Schadendorf D, Hassel JC, Akerley W, van den Eertwegh AJ, Lutzky J, Lorigan P, Vaubel JM, Linette GP, Hogg D, Ottensmeier CH, Lebbé C, Peschel C, Quirt I, Clark JI, Wolchok JD, Weber JS, Tian J, Yellin MJ, Nichol GM, Hoos A, Urba WJ (2010) Improved survival with ipilimumab in patients with metastatic melanoma. N Engl J Med 363(8):711-723.

https//doi.org/10.1056/NEJMoa1003466

21. Jia D, Li S, Li D, Xue H, Yang D, Liu Y (2018) Mining TCGA database for genes of prognostic value in glioblastoma microenvironment. Aging 10(4):592-605. https//doi.org/10.18632/aging.101415

22. Jiang P, Gu S, Pan D, Fu J, Sahu A, Hu X, Li Z, Traugh N, Bu X, Li B, Liu J, Freeman GJ, Brown MA, Wucherpfennig KW, Liu XS (2018) Signatures of T cell dysfunction and exclusion predict cancer immunotherapy response. Nat Med 24(10):1550-1558. https//doi.org/10.1038/s41591-018-0136-1

23. Korbecki J, Kojder K, Simińska D, Bohatyrewicz R, Gutowska I, Chlubek D, Baranowska-Bosiacka I (2020) CC chemokines in a tumor: a review of pro-cancer and anti-cancer properties of the ligands of receptors CCR1, CCR2, CCR3, and CCR4. Int J Mol Sci 21(21):8412. https//doi.org/10.3390/ijms21218412

24. Li T, Fu J, Zeng Z, Cohen D, Li J, Chen Q, Li B, Liu XS (2020) TIMER2.0 for analysis of tumor-infiltrating immune cells. Nucleic Acids Res 48(W1):W509-w514. https//doi.org/10.1093/nar/gkaa407

25. Li X, Zhang Q, Zhao L, Jiang L, Qi A, Wei Q, Song X, Wang L, Zhang L, Zhao Y, Lv X, Wei M, Zhao L (2020b) A Combined four-mRNA signature associated with lymphatic metastasis for prognosis of colorectal cancer. J Cancer 11(8):21392149. https//doi.org/10.7150/jca.38796

26. Lin W, Wu S, Chen X, Ye Y, Weng Y, Pan Y, Chen Z, Chen L, Qiu X, Qiu S (2020) Characterization of hypoxia signature to evaluate the tumor immune microenvironment and predict prognosis in glioma groups. Front Oncol 10:796. https//doi.org/10.3389/fonc.2020.00796

27. Liu X, Li Y, Qian Z, Sun Z, Xu K, Wang K, Liu S, Fan X, Li S, Zhang Z, Jiang T, Wang Y (2018) A radiomic signature as a non-invasive predictor of progression-free survival in patients with lower-grade gliomas. Neuroimage Clin 20:10701077. https//doi.org/10.1016/j.nicl.2018.10.014

28. Liu Z, Ravindranathan R, Li J, Kalinski P, Guo ZS, Bartlett DL (2016) CXCL11-Armed oncolytic poxvirus elicits potent antitumor immunity and shows enhanced therapeutic efficacy. Oncoimmunology 5(3):e1091554.

https//doi.org/10.1080/2162402x.2015.1091554

Page $14 / 27$ 
29. Louis DN, Perry A, Reifenberger G, von Deimling A, Figarella-Branger D, Cavenee WK, Ohgaki H, Wiestler OD, Kleihues P, Ellison DW (2016) The 2016 World Health Organization classification of tumors of the central nervous system: a summary. Acta Neuropathol 131(6):803-820. https//doi.org/10.1007/s00401-016-1545-1

30. Maru SV, Holloway KA, Flynn G, Lancashire CL, Loughlin AJ, Male DK, Romero IA (2008) Chemokine production and chemokine receptor expression by human glioma cells: role of CXCL10 in tumour cell proliferation. J Neuroimmunol 199(1-2):35-45. https//doi.org/10.1016/j.jneuroim.2008.04.029

31. Metsalu T, Vilo J (2015) Nucleic Acids Res 43(W1):W566-570. https//doi.org/10.1093/nar/gkv468. ClustVis: a web tool for visualizing clustering of multivariate data using Principal Component Analysis and heatmap

32. Miao YR, Zhang Q, Lei Q, Luo M, Xie GY, Wang H, Guo AY (2020) ImmuCellAl: a unique method for comprehensive T-Cell subsets abundance prediction and its application in cancer immunotherapy. Adv Sci (Weinh) 7(7):1902880. https//doi.org/10.1002/advs.201902880

33. Mitchem JB, Brennan DJ, Knolhoff BL, Belt BA, Zhu Y, Sanford DE, Belaygorod L, Carpenter D, Collins L, Piwnica-Worms D, Hewitt S, Udupi GM, Gallagher WM, Wegner C, West BL, Wang-Gillam A, Goedegebuure P, Linehan DC, DeNardo DG (2013) Targeting tumor-infiltrating macrophages decreases tumor-initiating cells, relieves immunosuppression, and improves chemotherapeutic responses. Cancer Res 73(3):1128-1141. https//doi.org/10.1158/0008-5472.can-12-2731

34. Molenaar RJ, Verbaan D, Lamba S, Zanon C, Jeuken JW, Boots-Sprenger SH, Wesseling P, Hulsebos TJ, Troost D, van Tilborg AA, Leenstra S, Vandertop WP, Bardelli A, van Noorden CJ, Bleeker FE (2014) The combination of IDH1 mutations and MGMT methylation status predicts survival in glioblastoma better than either IDH1 or MGMT alone. Neuro Oncol 16(9):1263-1273. https//doi.org/10.1093/neuonc/nou005

35. Mollica Poeta V, Massara M, Capucetti A, Bonecchi R (2019) Chemokines and chemokine receptors: new targets for cancer immunotherapy. Front Immunol 10:379. https//doi.org/10.3389/fimmu.2019.00379

36. Nagarsheth N, Wicha MS, Zou W (2017) Chemokines in the cancer microenvironment and their relevance in cancer immunotherapy. Nat Rev Immunol 17(9):559-572. https//doi.org/10.1038/nri.2017.49

37. Newman AM, Liu CL, Green MR, Gentles AJ, Feng W, Xu Y, Hoang CD, Diehn M, Alizadeh AA (2015) Robust enumeration of cell subsets from tissue expression profiles. Nat Methods 12(5):453-457. https//doi.org/10.1038/nmeth.3337

38. Omuro A, Vlahovic G, Lim M, Sahebjam S, Baehring J, Cloughesy T, Voloschin A, Ramkissoon SH, Ligon KL, Latek R, Zwirtes R, Strauss L, Paliwal P, Harbison CT, Reardon DA, Sampson JH (2018) Nivolumab with or without ipilimumab in patients with recurrent glioblastoma: results from exploratory phase I cohorts of CheckMate 143. Neuro Oncol 20(5):674-686. https//doi.org/10.1093/neuonc/nox208

39. Osipov A, Saung MT, Zheng L, Murphy AG (2019) Small molecule immunomodulation: the tumor microenvironment and overcoming immune escape. J Immunother Cancer 7(1):224. https//doi.org/10.1186/s40425-019-0667-0

40. Park MH, Lee JS, Yoon JH (2012) High expression of CX3CL1 by tumor cells correlates with a good prognosis and increased tumor-infiltrating CD8+ T cells, natural killer cells, and dendritic cells in breast carcinoma. J Surg Oncol 106(4):386-392. https//doi.org/10.1002/jso.23095

41. Qi C, Lei L, Hu J, Wang G, Liu J, Ou S (2021) T cell immune regulator 1 is a prognostic marker associated with immune infiltration in glioblastoma multiforme. Oncol Lett 21(4):252. https//doi.org/10.3892/ol.2021.12514

42. Ren F, Zhao Q, Huang L, Zheng Y, Li L, He Q, Zhang C, Li F, Maimela NR, Sun Z, Jia Q, Ping Y, Zhang Z, Chen X, Yue Y, Liu S, Cao L, Zhang Y (2019) The R132H mutation in IDH1 promotes the recruitment of NK cells through CX3CL1/CX3CR1 chemotaxis and is correlated with a better prognosis in gliomas. Immunol Cell Biol 97(5):457-469.

https//doi.org/10.1111/imcb.12225

43. Ritchie ME, Phipson B, Wu D, Hu Y, Law CW, Shi W, Smyth GK (2015) limma powers differential expression analyses for RNA-sequencing and microarray studies. Nucleic Acids Res 43(7):e47. https//doi.org/10.1093/nar/gkv007

44. Rizvi NA, Mazières J, Planchard D, Stinchcombe TE, Dy GK, Antonia SJ, Horn L, Lena H, Minenza E, Mennecier B, Otterson GA, Campos LT, Gandara DR, Levy BP, Nair SG, Zalcman G, Wolf J, Souquet PJ, Baldini E, Cappuzzo F, Chouaid C, Dowlati A, Sanborn R, Lopez-Chavez A, Grohe C, Huber RM, Harbison CT, Baudelet C, Lestini BJ, Ramalingam SS 
(2015) Activity and safety of nivolumab, an anti-PD-1 immune checkpoint inhibitor, for patients with advanced, refractory squamous non-small-cell lung cancer (CheckMate 063): a phase 2, single-arm trial. Lancet Oncol 16(3):257265. https//doi.org/10.1016/s1470-2045(15)70054-9

45. Ru B, Wong CN, Tong Y, Zhong JY, Zhong SSW, Wu WC, Chu KC, Wong CY, Lau CY, Chen I, Chan NW, Zhang J (2019) TISIDB: an integrated repository portal for tumor-immune system interactions. Bioinformatics 35(20):4200-4202. https//doi.org/10.1093/bioinformatics/btz210

46. Stupp R, Mason WP, van den Bent MJ, Weller M, Fisher B, Taphoorn MJ, Belanger K, Brandes AA, Marosi C, Bogdahn U, Curschmann J, Janzer RC, Ludwin SK, Gorlia T, Allgeier A, Lacombe D, Cairncross JG, Eisenhauer E, Mirimanoff RO (2005) Radiotherapy plus concomitant and adjuvant temozolomide for glioblastoma. N Engl J Med 352(10):987-996. https//doi.org/10.1056/NEJMoa043330

47. Thorsson V, Gibbs DL, Brown SD, Wolf D, Bortone DS, Ou Yang TH, Porta-Pardo E, Gao GF, Plaisier CL, Eddy JA, Ziv E, Culhane AC, Paull EO, Sivakumar IKA, Gentles AJ, Malhotra R, Farshidfar F, Colaprico A, Parker JS, Mose LE, Vo NS, Liu J, Liu Y, Rader J, Dhankani V, Reynolds SM, Bowlby R, Califano A, Cherniack AD, Anastassiou D, Bedognetti D, Mokrab Y, Newman AM, Rao A, Chen K, Krasnitz A, Hu H, Malta TM, Noushmehr H, Pedamallu CS, Bullman S, Ojesina Al, Lamb A, Zhou W, Shen H, Choueiri TK, Weinstein JN, Guinney J, Saltz J, Holt RA, Rabkin CS, Lazar AJ, Serody JS, Demicco EG, Disis ML, Vincent BG, Shmulevich I (2018) The immune landscape of cancer. Immunity 48(4):812-830. .e814. https//doi.org/10.1016/j.immuni.2018.03.023

48. Tokunaga R, Zhang W, Naseem M, Puccini A, Berger MD, Soni S, McSkane M, Baba H, Lenz HJ (2018) CXCL9, CXCL10, CXCL11/CXCR3 axis for immune activation-a target for novel cancer therapy. Cancer Treat Rev 63:40-47. https//doi.org/10.1016/j.ctrv.2017.11.007

49. Uhlén M, Björling E, Agaton C, Szigyarto CA, Amini B, Andersen E, Andersson AC, Angelidou P, Asplund A, Asplund C, Berglund L, Bergström K, Brumer H, Cerjan D, Ekström M, Elobeid A, Eriksson C, Fagerberg L, Falk R, Fall J, Forsberg M, Björklund MG, Gumbel K, Halimi A, Hallin I, Hamsten C, Hansson M, Hedhammar M, Hercules G, Kampf C, Larsson K, Lindskog M, Lodewyckx W, Lund J, Lundeberg J, Magnusson K, Malm E, Nilsson P, Odling J, Oksvold P, Olsson I, Oster E, Ottosson J, Paavilainen L, Persson A, Rimini R, Rockberg J, Runeson M, Sivertsson A, Sköllermo A, Steen J, Stenvall M, Sterky F, Strömberg S, Sundberg M, Tegel H, Tourle S, Wahlund E, Waldén A, Wan J, Wernérus H, Westberg J, Wester K, Wrethagen U, Xu LL, Hober S, Pontén F (2005) A human protein atlas for normal and cancer tissues based on antibody proteomics. Mol Cell Proteomics 4(12):1920-1932. https//doi.org/10.1074/mcp.M500279-MCP200

50. Uhlen M, Oksvold P, Fagerberg L, Lundberg E, Jonasson K, Forsberg M, Zwahlen M, Kampf C, Wester K, Hober S, Wernerus H, Björling L, Ponten F (2010) Towards a knowledge-based Human Protein Atlas. Nat Biotechnol 28(12):1248-1250. https//doi.org/10.1038/nbt1210-1248

51. Ulloa-Montoya F, Louahed J, Dizier B, Gruselle O, Spiessens B, Lehmann FF, Suciu S, Kruit WH, Eggermont AM, Vansteenkiste J, Brichard VG (2013) Predictive gene signature in MAGE-A3 antigen-specific cancer immunotherapy. J Clin Oncol 31(19):2388-2395. https//doi.org/10.1200/jco.2012.44.3762

52. Wang Y, Qian T, You G, Peng X, Chen C, You Y, Yao K, Wu C, Ma J, Sha Z, Wang S, Jiang T (2015) Localizing seizuresusceptible brain regions associated with low-grade gliomas using voxel-based lesion-symptom mapping. Neuro Oncol 17(2):282-288. https//doi.org/10.1093/neuonc/nou130

53. Wu T, Dai Y (2017) Tumor microenvironment and therapeutic response. Cancer Lett 387:61-68. https//doi.org/10.1016/j.canlet.2016.01.043

54. Wu X, Sun A, Yu W, Hong C, Liu Z (2020) CXCL10 mediates breast cancer tamoxifen resistance and promotes estrogendependent and independent proliferation. Mol Cell Endocrinol 512:110866. https//doi.org/10.1016/j.mce.2020.110866

55. Xu L, Deng C, Pang B, Zhang X, Liu W, Liao G, Yuan H, Cheng P, Li F, Long Z, Yan M, Zhao T, Xiao Y, Li X (2018) TIP: a web server for eesolving tumor immunophenotype profiling. Cancer Res 78(23):6575-6580.

https//doi.org/10.1158/0008-5472.can-18-0689

Page $16 / 27$ 
56. Yang L, Jackson E, Woerner BM, Perry A, Piwnica-Worms D, Rubin JB (2007) Blocking CXCR4-mediated cyclic AMP suppression inhibits brain tumor growth in vivo. Cancer Res 67(2):651-658. https//doi.org/10.1158/0008-5472.can-062762

57. Yang XL, Liu KY, Lin FJ, Shi HM, Ou ZL (2017) CCL28 promotes breast cancer growth and metastasis through MAPKmediated cellular anti-apoptosis and pro-metastasis. Oncol Rep 38(3):1393-1401.

https//doi.org/10.3892/or.2017.5798

58. Yin W, Jiang X, Tan J, Xin Z, Zhou Q, Zhan C, Fu X, Wu Z, Guo Y, Jiang Z, Ren C, Tang G (2020) Development and validation of a tumor mutation burden-related immune prognostic model for lower-grade glioma. Front Oncol 10:1409. https//doi.org/10.3389/fonc.2020.01409

59. Yoshihara K, Shahmoradgoli M, Martínez E, Vegesna R, Kim H, Torres-Garcia W, Treviño V, Shen H, Laird PW, Levine DA, Carter SL, Getz G, Stemke-Hale K, Mills GB, Verhaak RG (2013) Inferring tumour purity and stromal and immune cell admixture from expression data. Nat Commun 4:2612. https//doi.org/10.1038/ncomms3612

60. Zhang Y, Ni S, Huang B, Wang L, Zhang X, Li X, Wang H, Liu S, Hao A, Li X (2015) Overexpression of SCLIP promotes growth and motility in glioblastoma cells. Cancer Biol Ther 16(1):97-105.

https//doi.org/10.4161/15384047.2014.987037

61. Zhao Z, Meng F, Wang W, Wang Z, Zhang C, Jiang T (2017) Comprehensive RNA-seq transcriptomic profiling in the malignant progression of gliomas. Sci Data 4:170024. https//doi.org/10.1038/sdata.2017.24

62. Zhou Z, Zhang J, Xu C, Yang J, Zhang Y, Liu M, Shi X, Li X, Zhan H, Chen W, McNally LR, Fung KM, Luo W, Houchen CW, He Y, Zhang C, Li M (2021) An integrated model of N6-methyladenosine regulators to predict tumor aggressiveness and immune evasion in pancreatic cancer. EBioMedicine 65:103271. https//doi.org/10.1016/j.ebiom.2021.103271

\section{Table 1}

Table 1 Clinical data of five independent datasets

\begin{tabular}{|c|c|c|c|c|c|}
\hline Variables & $\begin{array}{l}\text { TCGA- } \\
\text { LGG }\end{array}$ & $\begin{array}{c}\text { CGGA mRNA- } \\
325 \\
\end{array}$ & $\begin{array}{c}\text { CGGA mRNA- } \\
693 \\
\end{array}$ & $\begin{array}{c}\text { CGGA mRNA } \\
\text { array_301 }\end{array}$ & GSE16011 \\
\hline \multicolumn{6}{|l|}{ Gender } \\
\hline Female & 226 & 65 & 185 & 68 & 36 \\
\hline Male & 269 & 105 & 235 & 90 & 67 \\
\hline NA & 0 & 0 & 0 & 0 & 0 \\
\hline \multicolumn{6}{|l|}{ Age } \\
\hline$<=40$ & 242 & 95 & 211 & 90 & 39 \\
\hline$>40$ & 253 & 75 & 208 & 66 & 64 \\
\hline NA & 0 & 0 & 1 & 2 & 0 \\
\hline \multicolumn{6}{|l|}{ WHO grade } \\
\hline II & 241 & 96 & 172 & 105 & 23 \\
\hline III & 254 & 74 & 248 & 53 & 80 \\
\hline \multicolumn{6}{|l|}{ IDH-1 status } \\
\hline Wild type & 104 & 44 & 94 & 53 & 38 \\
\hline Mutant type & 372 & 125 & 288 & 104 & 46 \\
\hline NA & 19 & 1 & 38 & 1 & 19 \\
\hline \multicolumn{6}{|l|}{$\begin{array}{l}1 \mathrm{p} 19 \mathrm{q} \text { codel } \\
\text { status }\end{array}$} \\
\hline Codeletion & 234 & 55 & 125 & 16 & 37 \\
\hline Non-codeletion & 258 & 113 & 257 & 33 & 38 \\
\hline NA & 3 & 2 & 38 & 109 & 28 \\
\hline \multicolumn{6}{|l|}{ Survival status } \\
\hline Alive & 364 & 82 & 223 & 85 & 17 \\
\hline Death & 131 & 88 & 197 & 73 & 86 \\
\hline Total & 495 & 170 & 420 & 158 & 103 \\
\hline
\end{tabular}




\section{Figures}

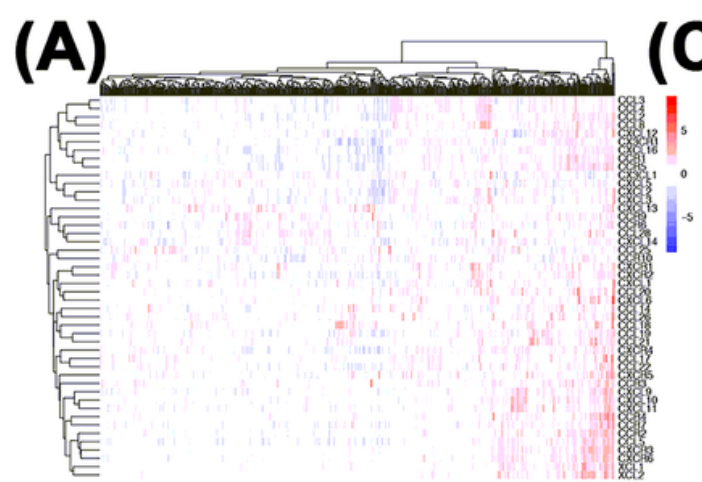

(C)

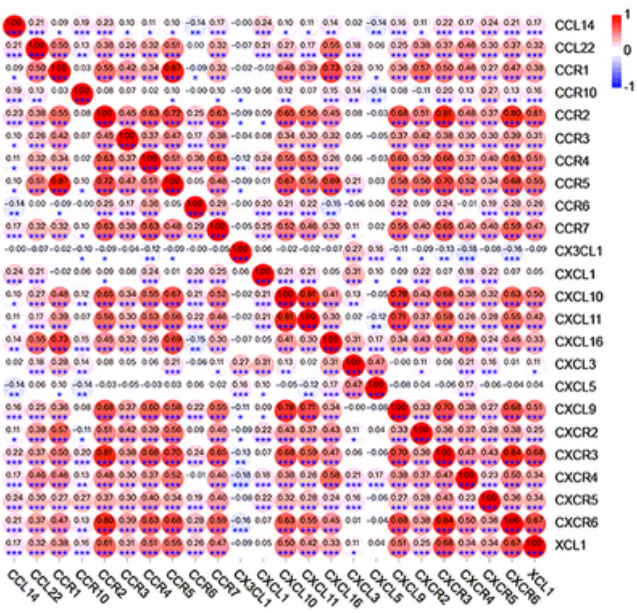

(D)
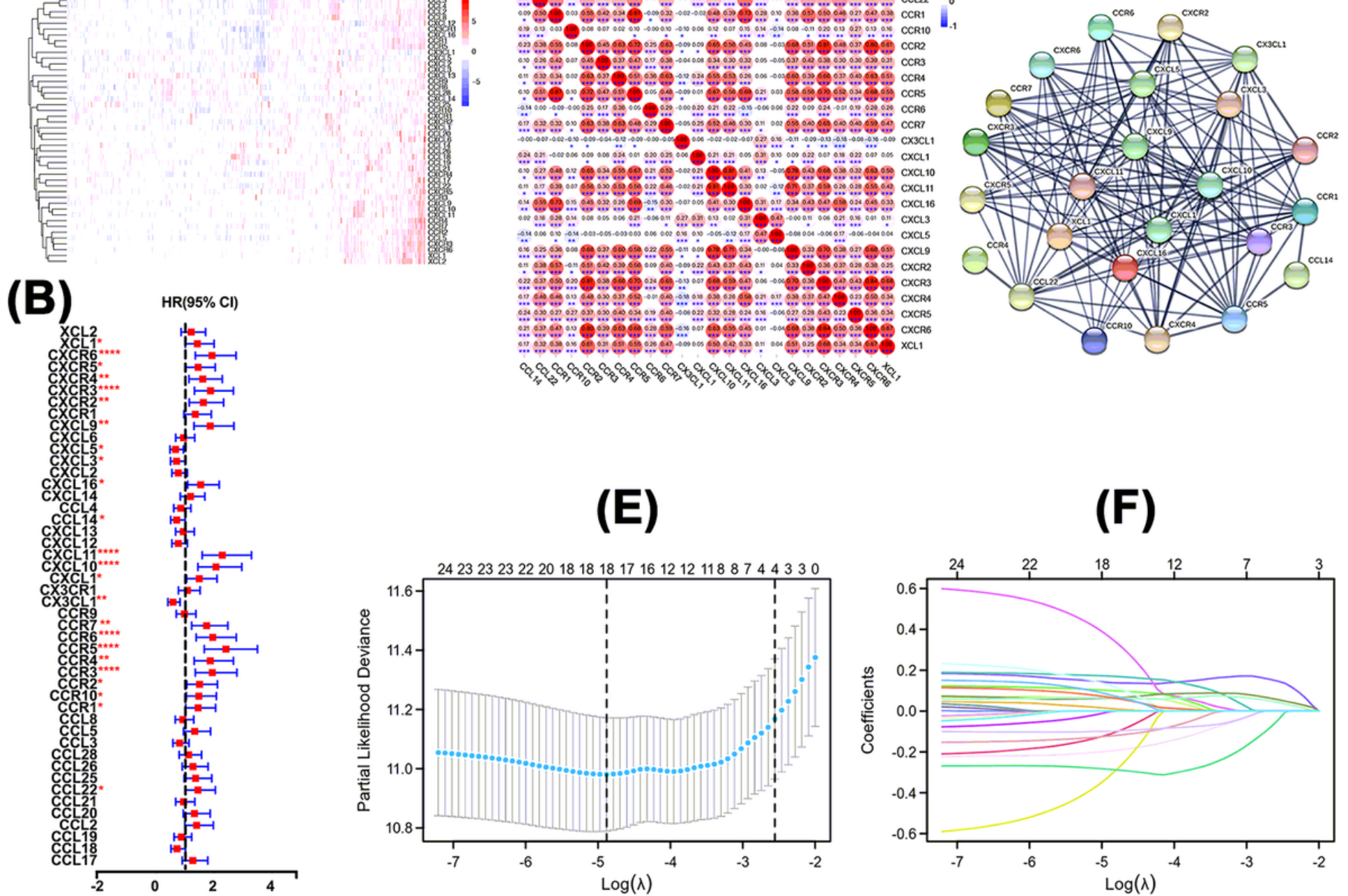

(E)

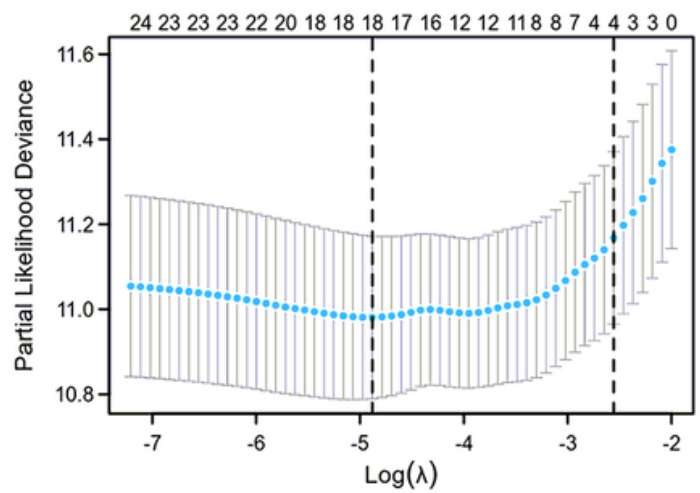

(F)

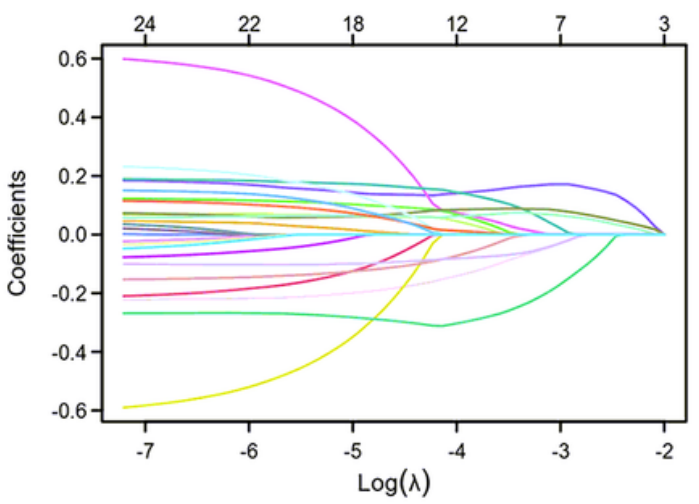

Figure 1

Candidate genes identification and a risk score model construction. A An expression heatmap of 46 chemokines and chemokine receptors in the TCGA-LGG dataset. B Univariate analysis identified 24 prognosis-related genes. C A correlation heatmap showed that most of these prognosis-related genes positively correlated with others. D A PPI network consisting of 24 nodes and 144 edges to reflect the prognosis-related genes. E,F The LASSO regression predictive formula (lambda.1-SE criteria) was used to identify the accurate prognosis-related genes $(E)$ and their coefficients $(F)$. The red "*" stands for statistical significance, *: $P<0.05 ; * \star: P<0.01 ; * \star *: P<0.0001 ; * \star \star *: P<0.0001$ 
(A)

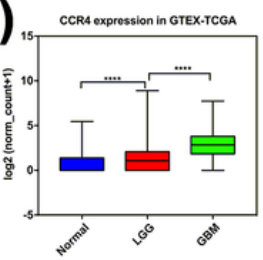

(E)

low grade glioma
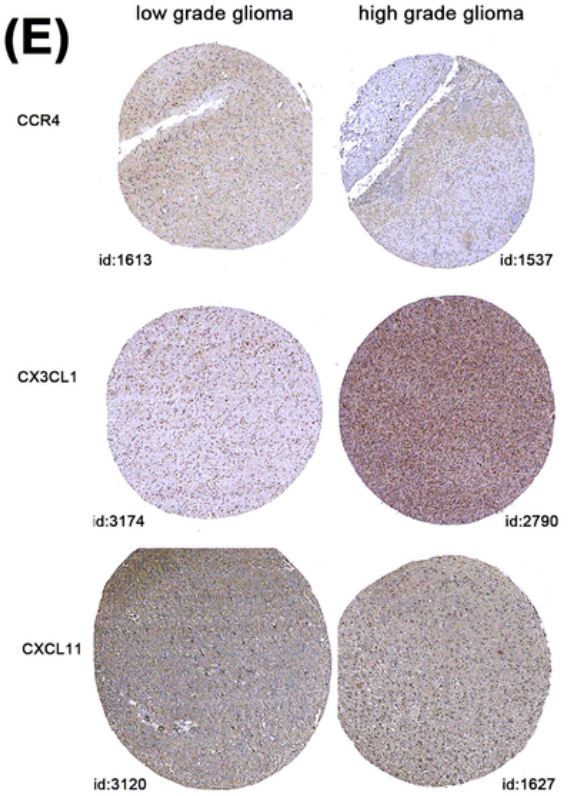

(B)

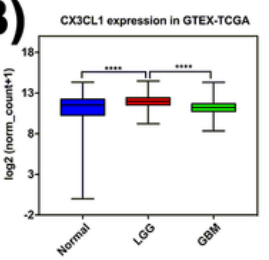

(C)

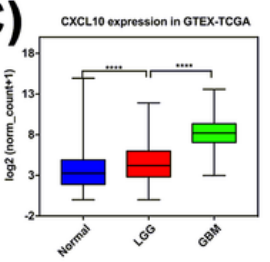

(D)

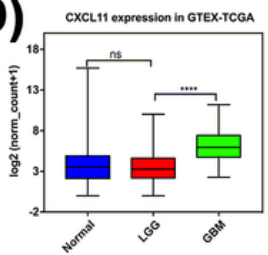

(F)
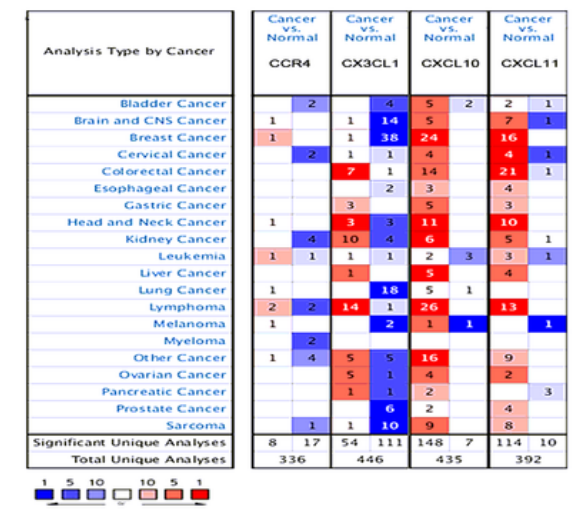

(G)
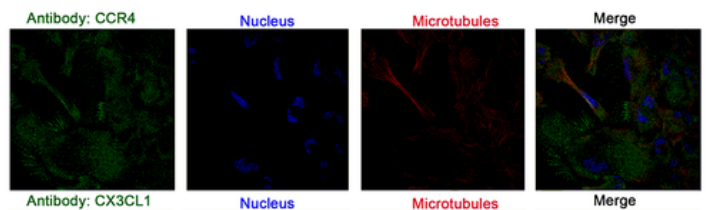
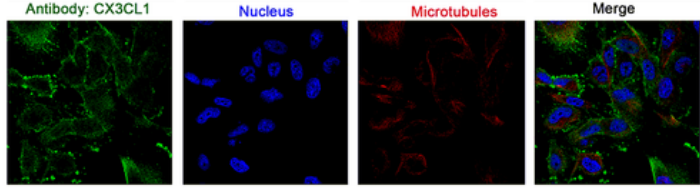

CXCL10

CXCL11
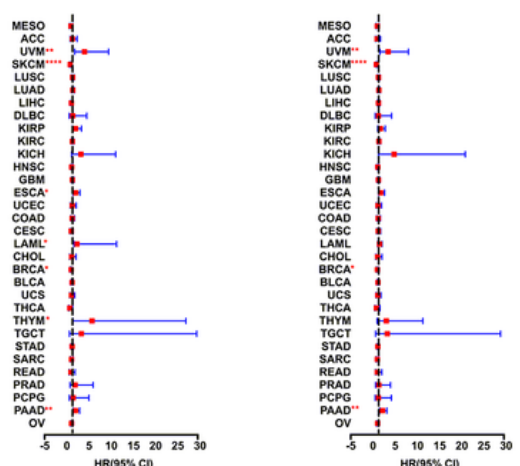

Figure 2

Multi-omics analyses of the genes in the risk score model. A-D High expression of CCR4 (A), CX3CL1 (B), CXCL10 (C) and CXCL11 (D) was found in glioma samples compared with normal brain tissues in transcriptomic levels by GTEX-TCGA database. E Protein levels exploration in HPA database. A weak intensity of CCR4 was found in low grade glioma and high grade glioma samples. CX3CL1 was found to be moderate and strong intensity staining in glioma samples. Weak and moderate intensity staining of CXCL11 were found in glioma tissues. F Expression of CCR4, CX3CL1, CXCL10 and CXCL11 in different types of human cancers with defined thresholds $p$ value 0.05 and fold change 1.0 in the Oncomine database, the cell number represents the dataset number that meets all of the thresholds with the color blue for under-expression and color red for over-expression. G Plasma membrane immunofluorescence staining of CCR4 and CX3CL1 in glioma U251 cell line. H Univariate Cox regression analyses of CCR4, CX3CL1, CXCL10 and CXCL11 in pan-cancer dataset showed different prognostic value in multiple human cancers. *: $p<0.05$; **: $p<0.01 ; * \star *: p<0.001 ; * \star \star *: p<0.0001$ 
(A)

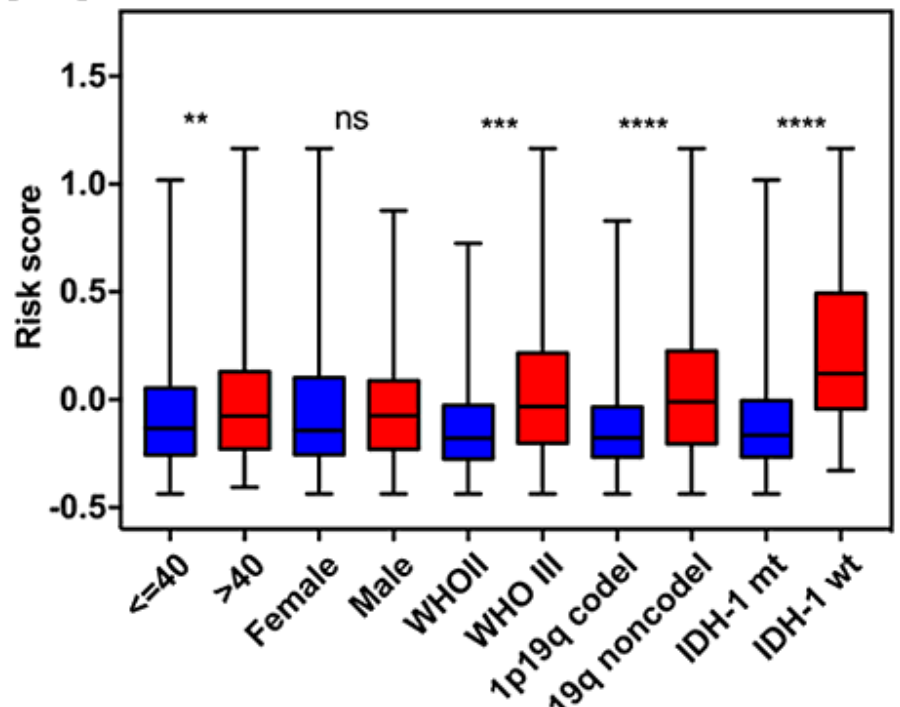

(C) CGGA mRNÂ array_301

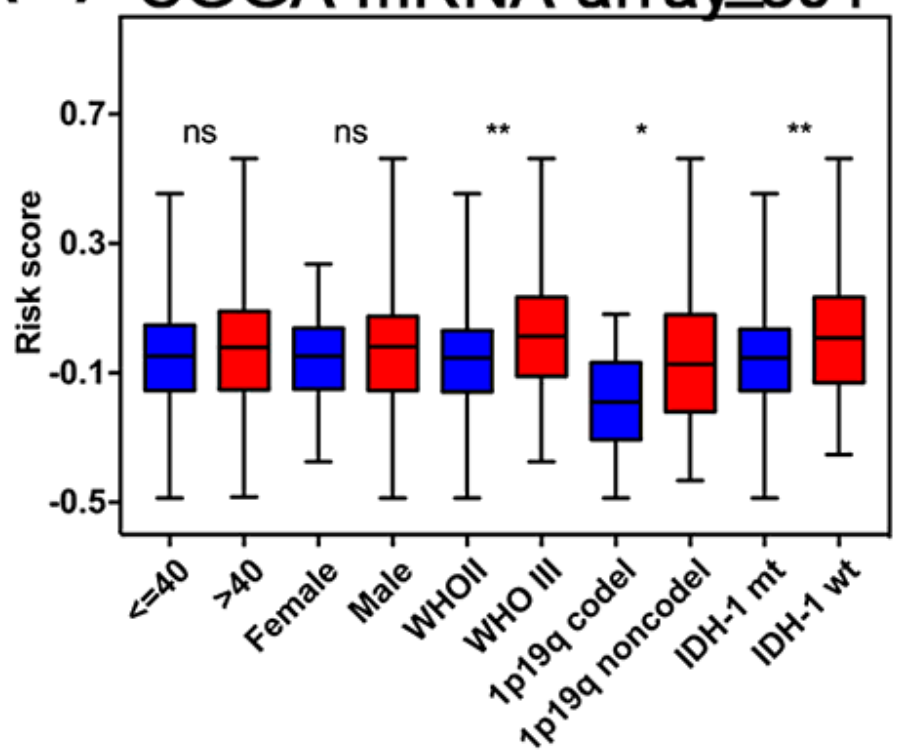

(B) CGGA mRNA-325

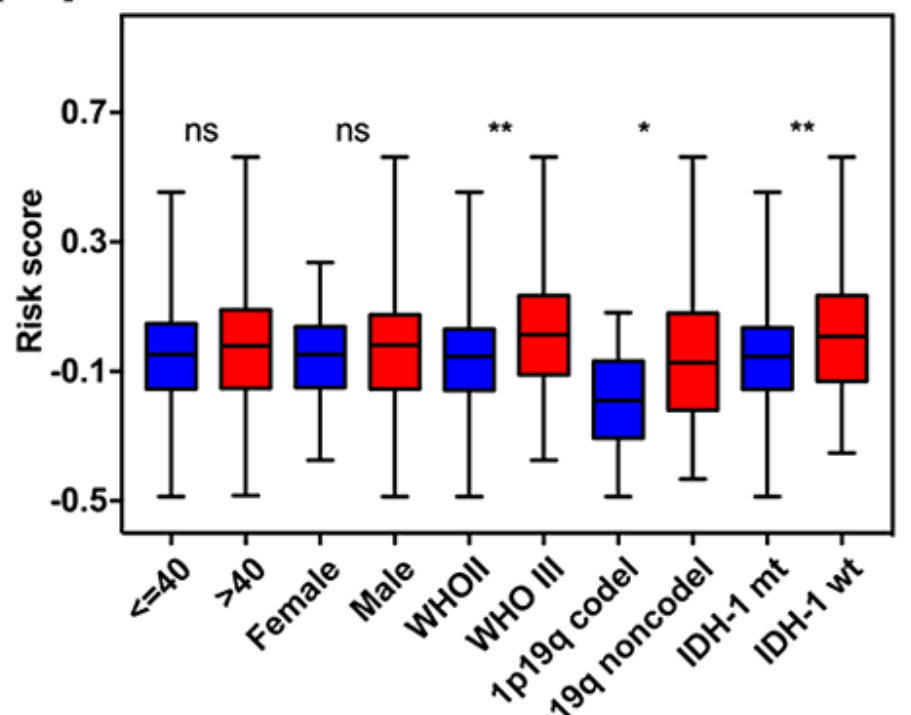

(D) CGGA mRिNA-693

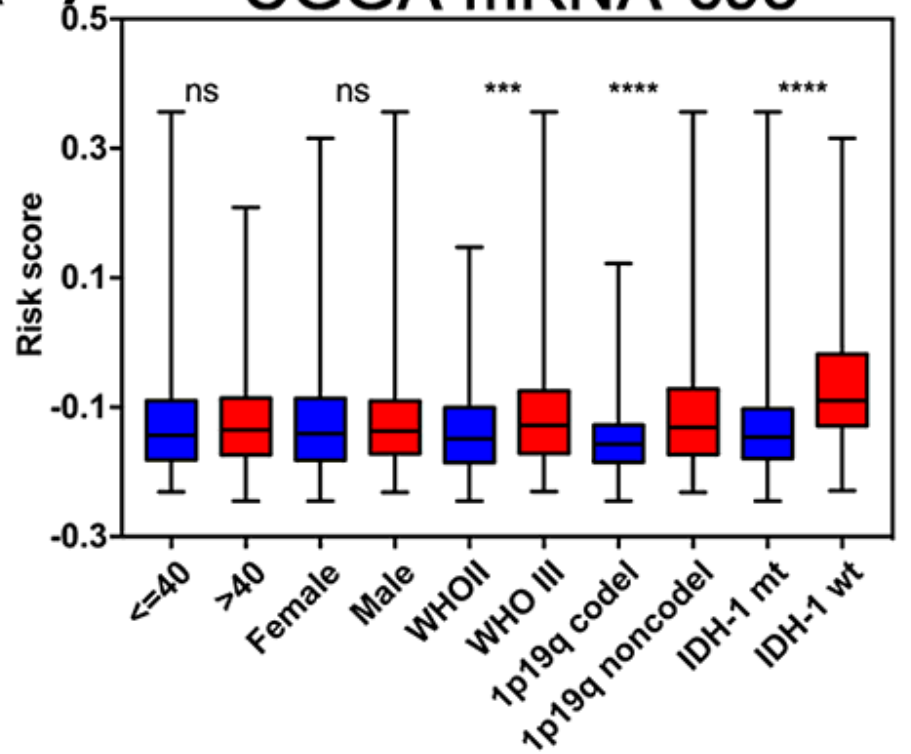

Figure 3

High-risk scores predict malignant phenotypes of LGG patients. A In the TCGA-LGG dataset, higher-risk scores are found in WHO III grade gliomas, and LGG patients with IDH-1 wild type or 1p19q non-codeletion type have higher risk scores. B-D LGG patients with higher risk scores in the CGGA mRNA-325 (B), CGGA mRNA array-301(C) and CGGA mRNA-693 datasets (D) have higher WHO grades and more malignant phenotypes. *: $p<0.05$; $* *$ : $p<0.01$; ***: $p<0.001 ; * \star \star *: p<0.0001$; ns: no statistical significance. IDH: isocitrate dehydrogenase; mt: mutation type; wt: wild type 
(A)
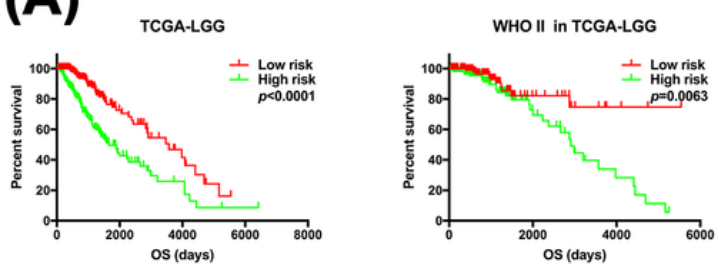

(B)

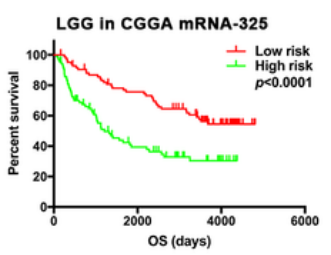

(C)

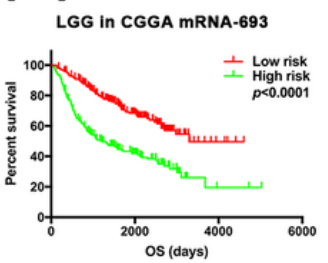

(D)

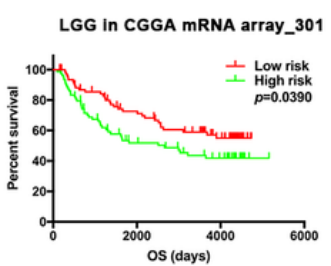

(E)
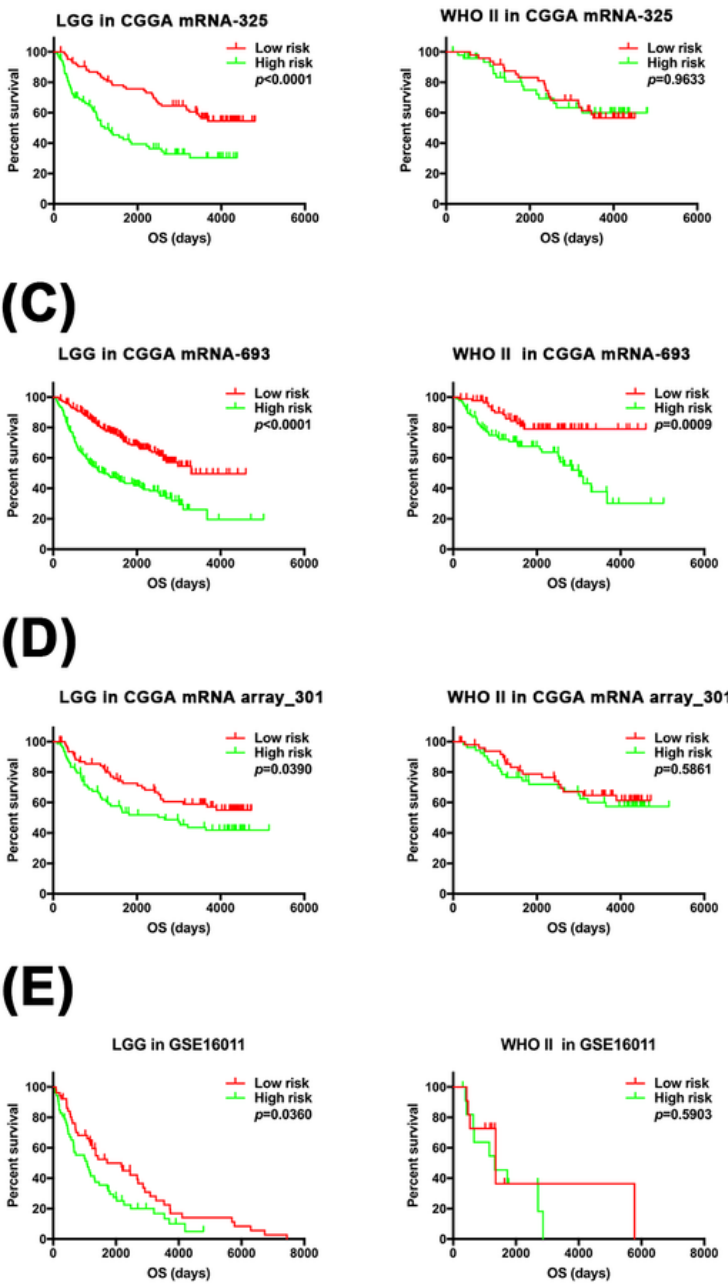
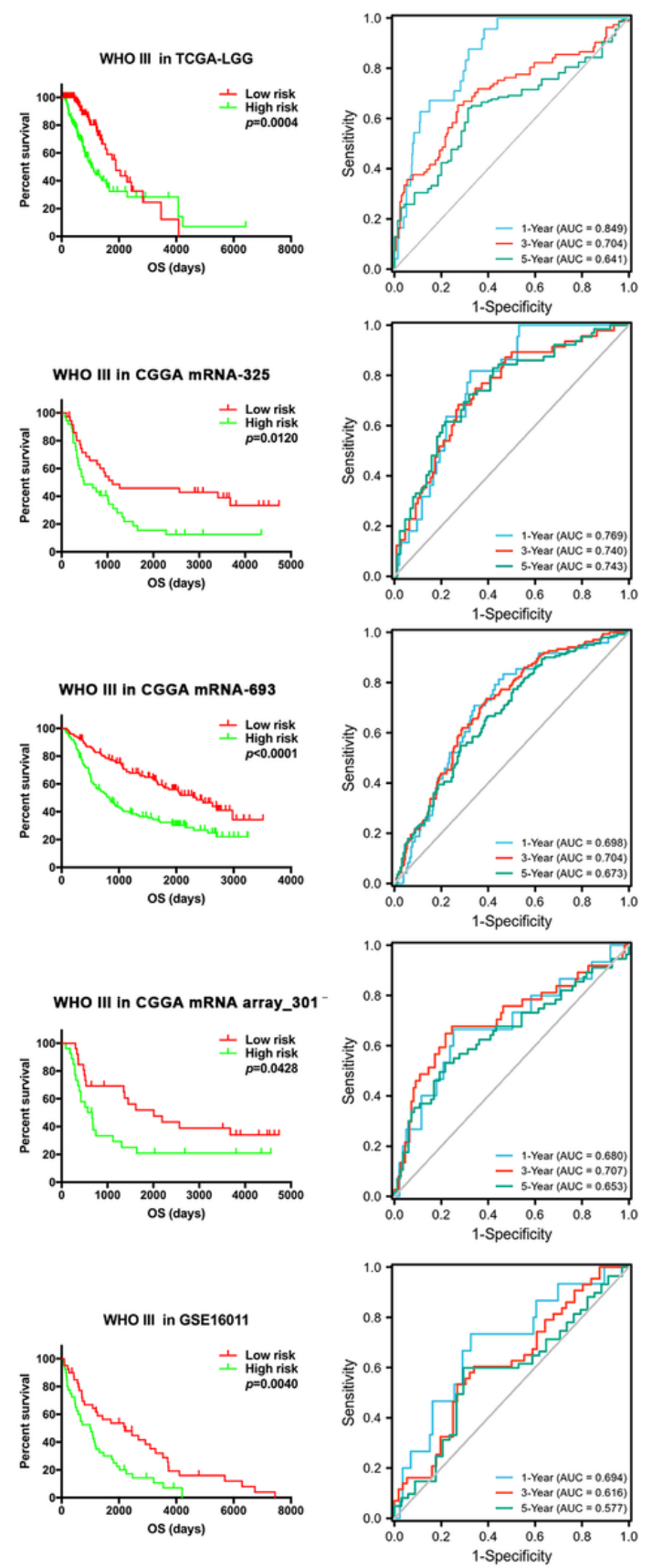

Figure 4

Survival analyses and time-dependent ROC curves for the risk score model for OS in the training dataset and the validation datasets. A In the TCGA-LGG dataset, the entire LGG patients, WHO grade II and WHO grade III glioma patients with high-risk scores have shorter OS. ROC curves were used to assess the predictive accuracy for 1-, 3- and 5-year OS. B-E High-risk score can predict worse prognosis for all LGG patients and WHO grade III glioma patients, but the difference for WHO grade II glioma patients in the CGGA mRNA-325 (B), mRNA array_301 (D) and GSE16011 (E) datasets were not significant. ROC: receiver operator characteristic; AUC: area under the curve 

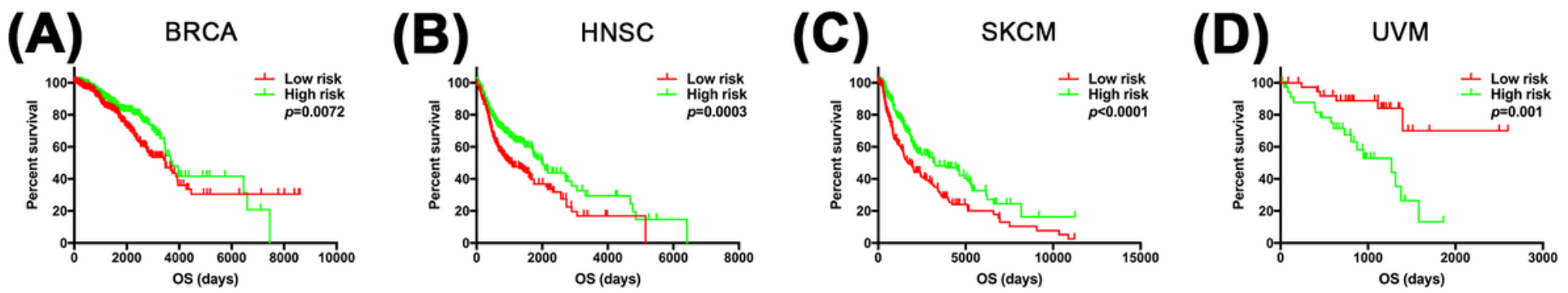

Figure 5

Validation of the four-gene risk score model in multi-cancers. A-D Kaplan-Meier curves indicated that high-risk scores in BRCA (A), HNSC (B) and SKCM (C) patients presented a better prognosis, but UVM (D) patients with high-risk score had a worse prognosis. BRCA: breast invasive carcinoma; HNSC: head and neck squamous carcinoma; SKCM: skin and cutaneous melanoma; UVM: uveal melanoma

(A)
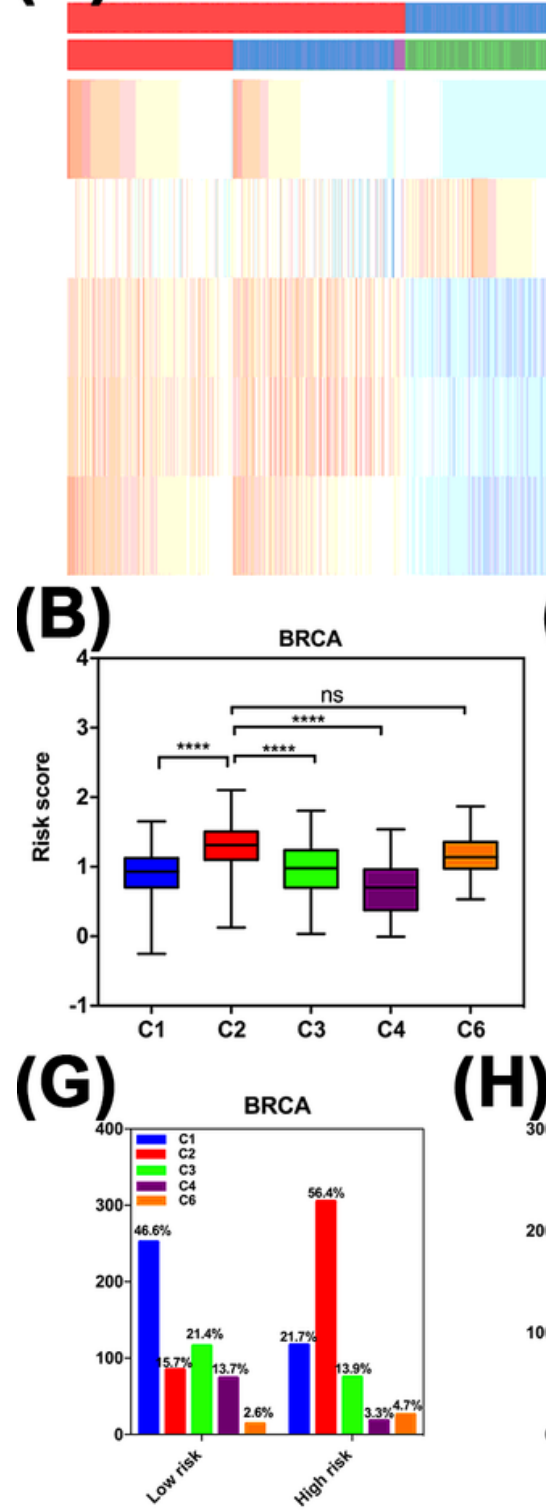

(H)
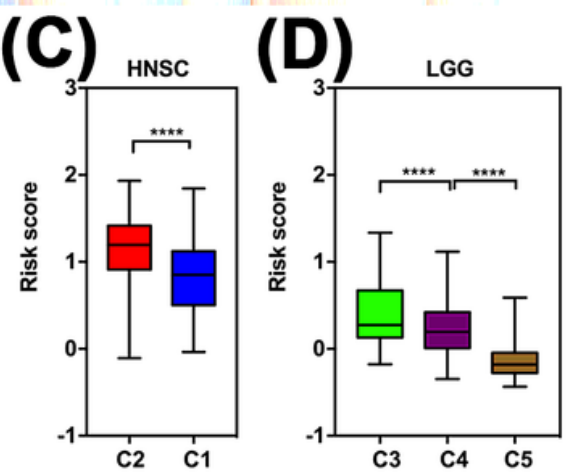

(l)
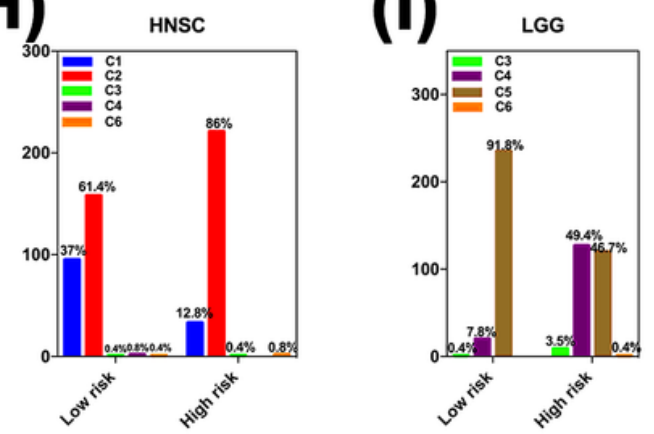

Immune subtype

Cancer type

CCR4 3

$\begin{array}{ll}\text { CX3CL1 } & 1\end{array}$

CXCL10

$\begin{array}{ll} & -1 \\ \text { CXCL11 } & -2 \\ \text { Risk score } & -3\end{array}$

Immune subtype

IFN-gamma Dominant (Immune C2) Immunologically Quiet (Immune C5)

Inflammatory (Immune C3)

Lymphocyte Depleted (Immune C4)

TGF-beta Dominant (Immune C6)

Wound Healing (Immune C1)

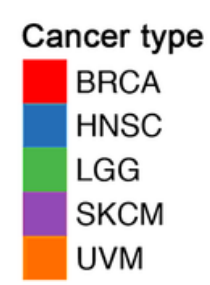

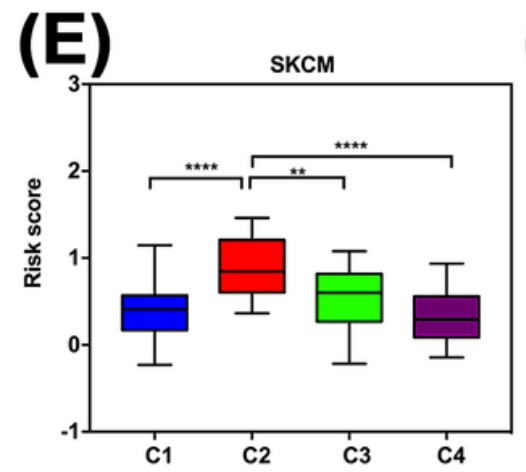
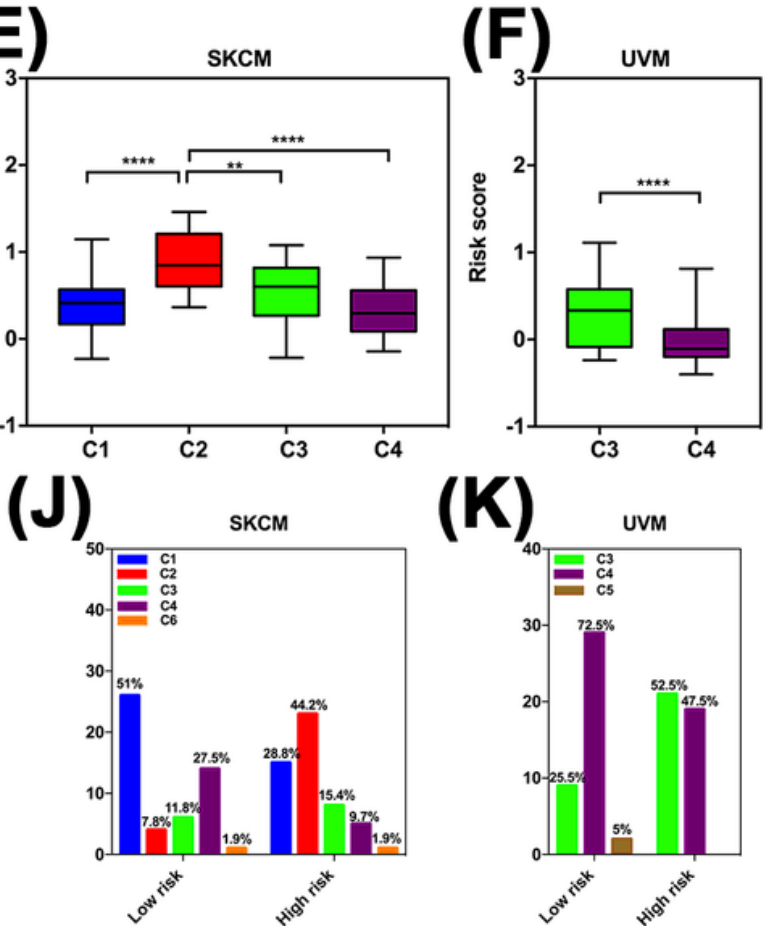

Figure 6 
Comparison of risk scores and immune subtypes among five TCGA cancers. A comparable heatmap showing the risk scores and CCR4, CX3CL1, CXCL10 and CXCL11 expression between different immune subtypes in five TCGA cancers (A). B-F The risk score distribution among different immune subtypes among five TCGA cancers showed that LGG (D) and UVM (F) patients in the C3 type (inflammatory) had higher risk scores, and patients with BRCA (B), HNSC (C) and SKCM (E) who were in the C2 type (IFN-gamma dominant) had higher risk scores. G-K The composition of immune subtypes in five TCGA cancers between low- and high-risk score groups. BRCA: breast invasive carcinoma; HNSC: head and neck squamous

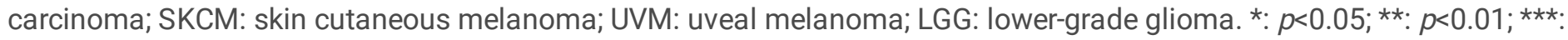
$p<0.001 ; * * \star *$ : $p<0.0001$; ns: no statistical significance
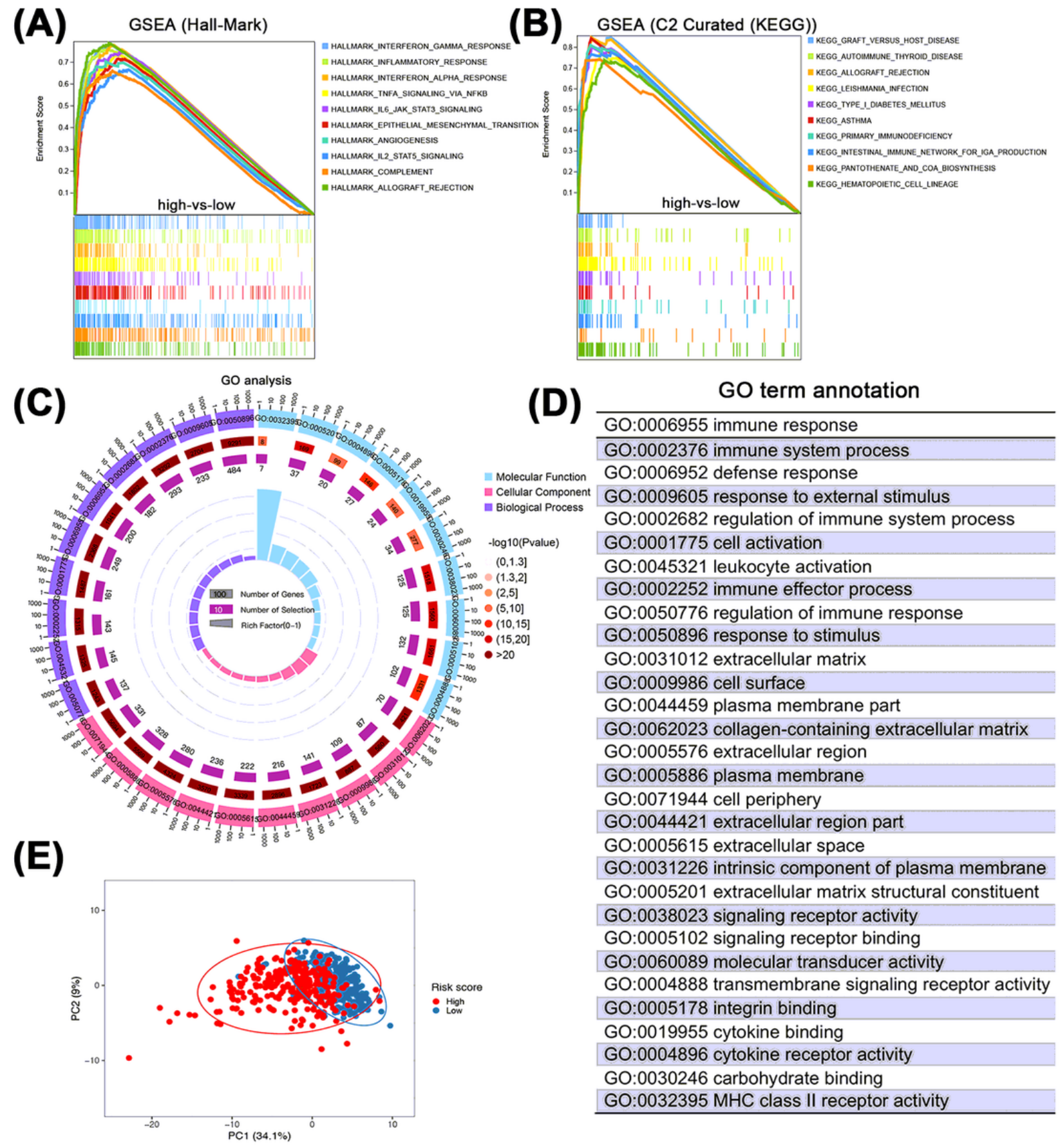

Figure 7

Functional enrichment analysis and PCA of high- and low-risk score groups. A,B GSEA showed that genes in the high-risk score groups were mainly enriched in immune-related pathways based on different gene sets, hallmark gene sets $(\mathbf{A})$ and curated gene sets (KEGG subset of canonical pathways)(B). C,D GO analysis showed that DEGs were mainly enriched in 
immune response, immune system process and defense response. The outermost ring represented the classification of enrichment, and different colors represent different classifications; The second outer ring represented the number of the classification in background genes and the $p$ value. The more genes there were, the longer the bar, and the smaller the $p$ value, the redder was the color. The third circle represents the total number of foreground genes. The fourth circle shows the enrichment factor value of each classification (number of foreground genes divided by number of background genes in this classification). E PCA showed that low- and high-risk groups were generally distributed in different directions based on immune-related genes. GSEA: gene set enrichment analysis; GO: gene ontology; KEGG: Kyoto Encyclopedia of Genes and Genomes; DEGs: differentially expressed genes; PCA: principal components analysis
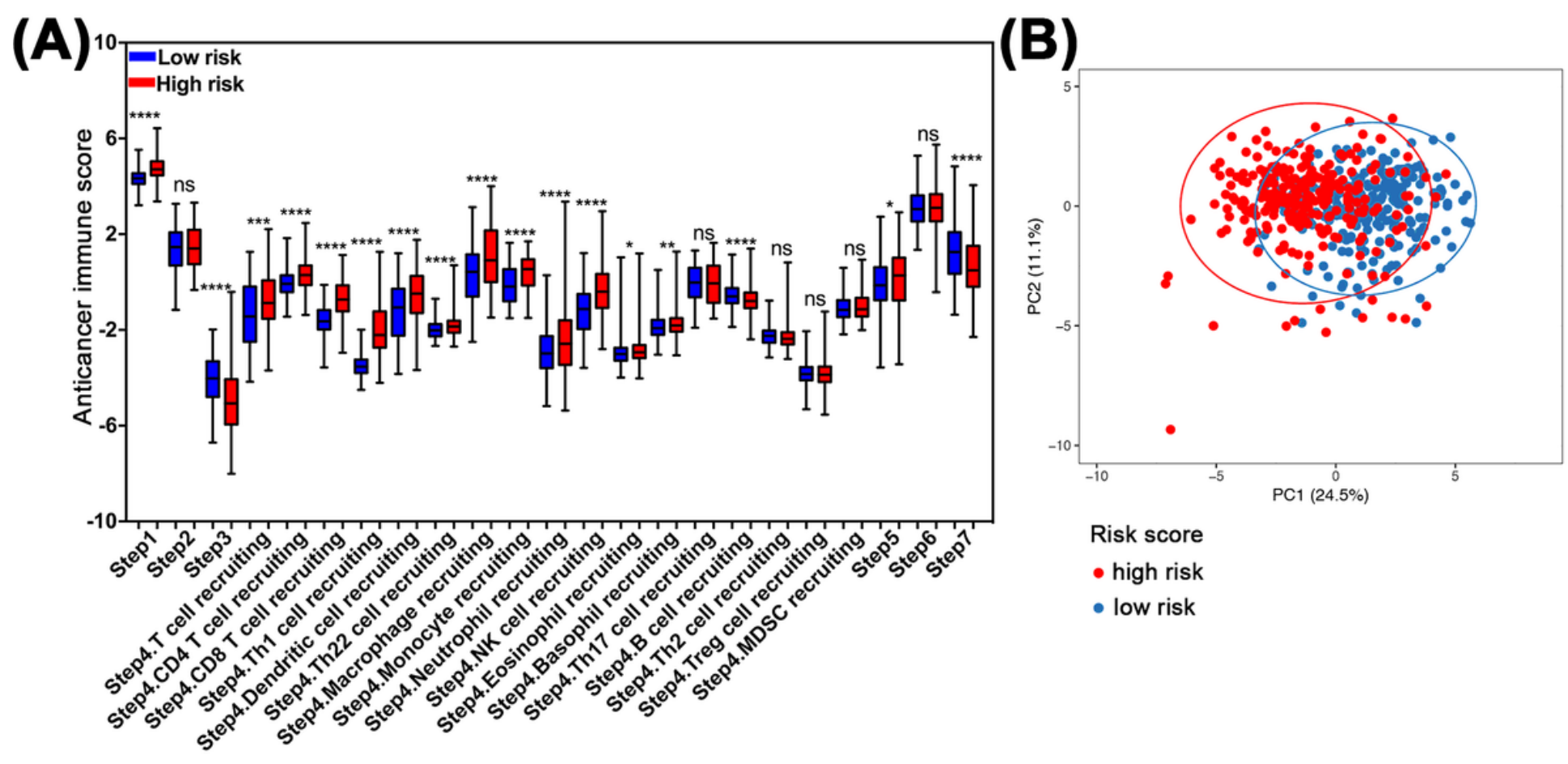

Risk score

- high risk

- low risk

Figure 8

The different anticancer immune activities between high- and low-risk score groups. A The different anticancer immune activity scores between high- and low-risk score groups. B PCA showed that anticancer immune activity scores in high- and low-risk score groups were generally distributed in different directions. $*$ : $p<0.05$; $* *: p<0.01 ; * \star \star: ~ p<0.001$; ****: $p<0.0001$; ns: no statistical significance; PCA: principal components analysis 

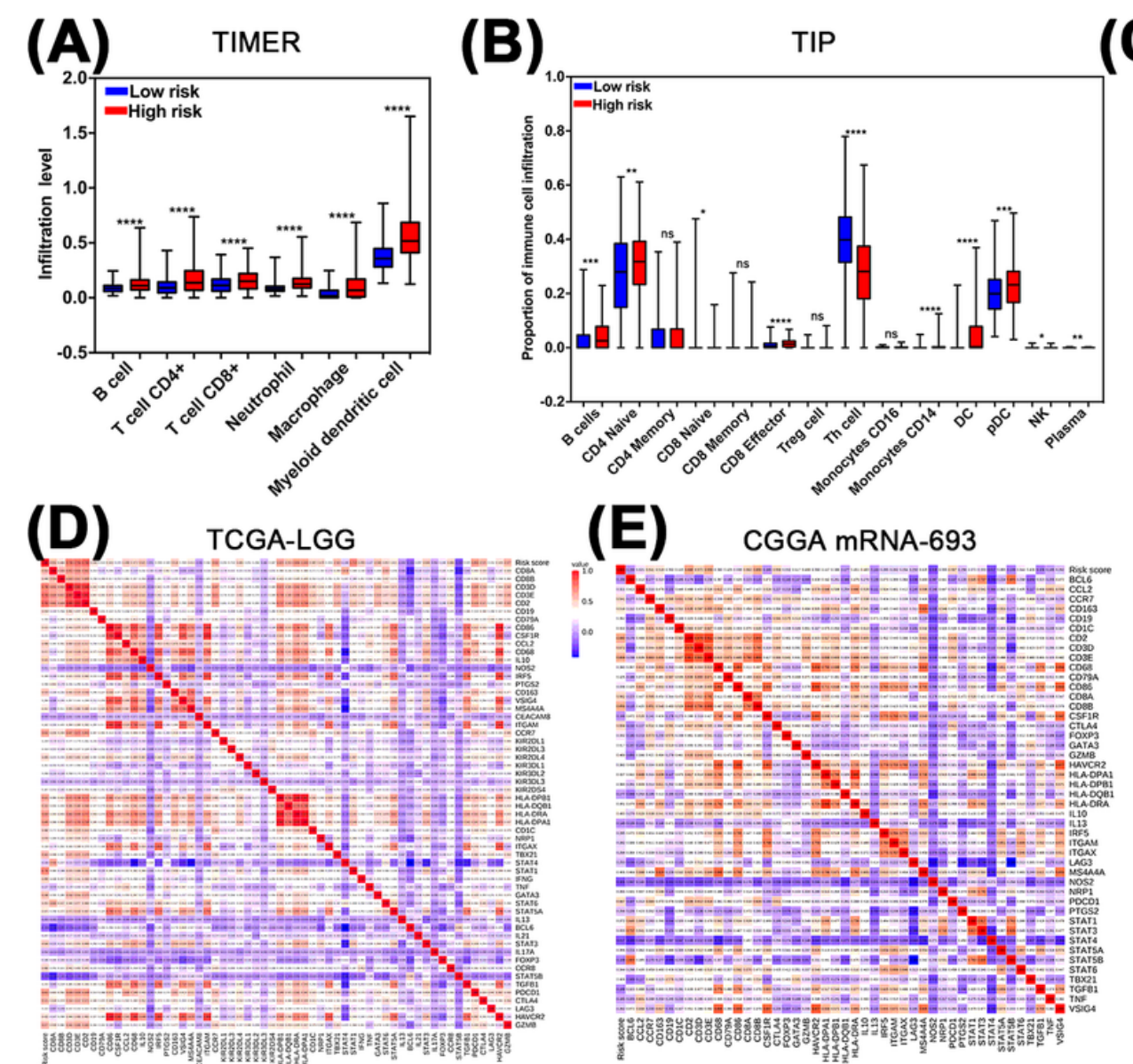

(C)
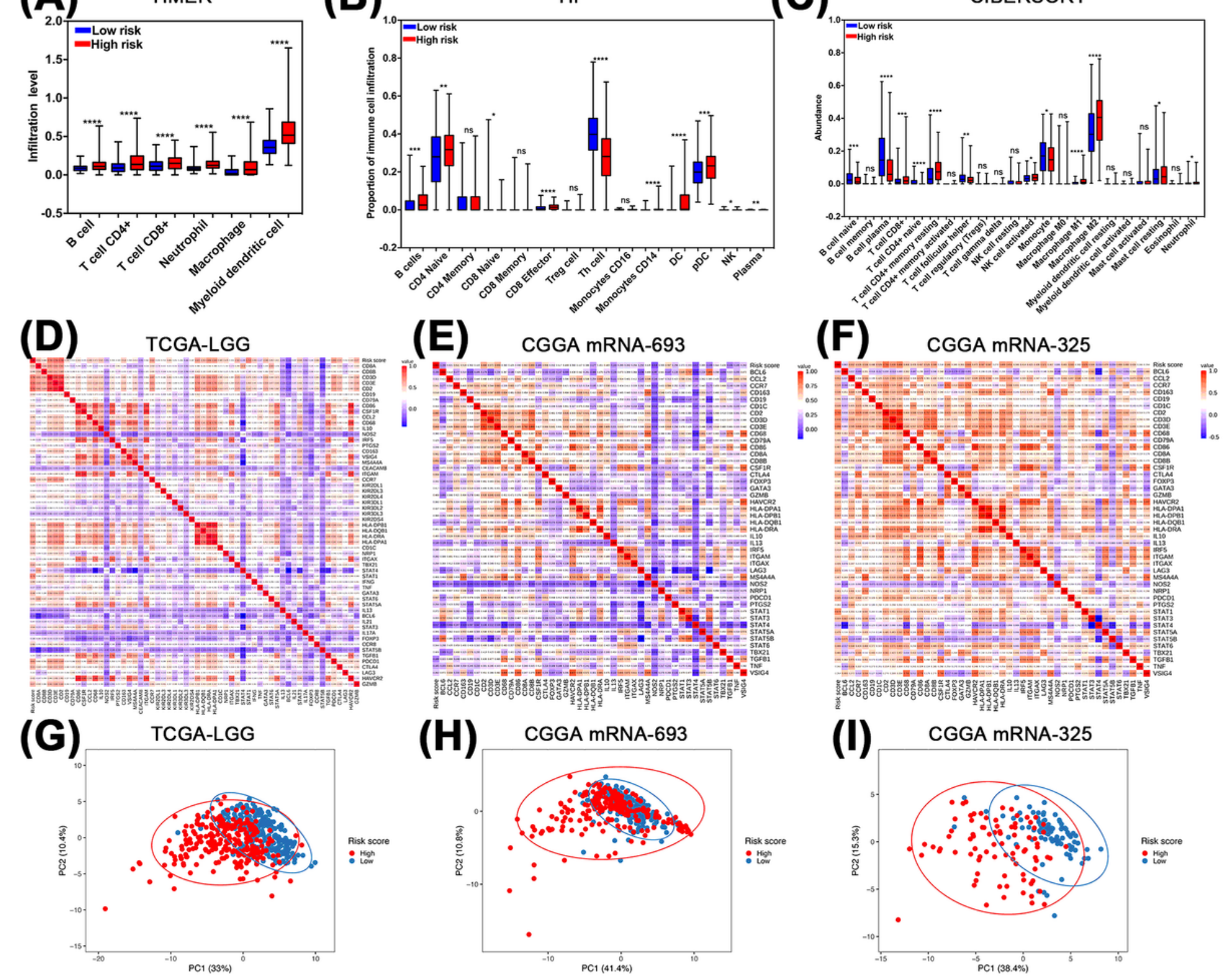

Figure 9

Three algorithms indicate different immune cell infiltration levels between high- and low-risk score groups. A-C TIMER (A), TIP (B) and CIBERSORT (C) algorithms analyses showed different immune cell infiltration between the low- and high-risk groups. D-F The correlation analyses between 57-gene markers expression and four-gene risk scores in the TCGA-LGG (D), CGGA mRNA-693 (E) and CGGA mRNA-325 (F) datasets. G-I PCA showed that in three independent datasets, immune gene

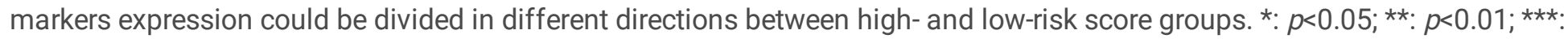
$p<0.001$; $* \star \star *: p<0.0001$; PCA: principal components analysis; ns: no statistical significance 
(A)

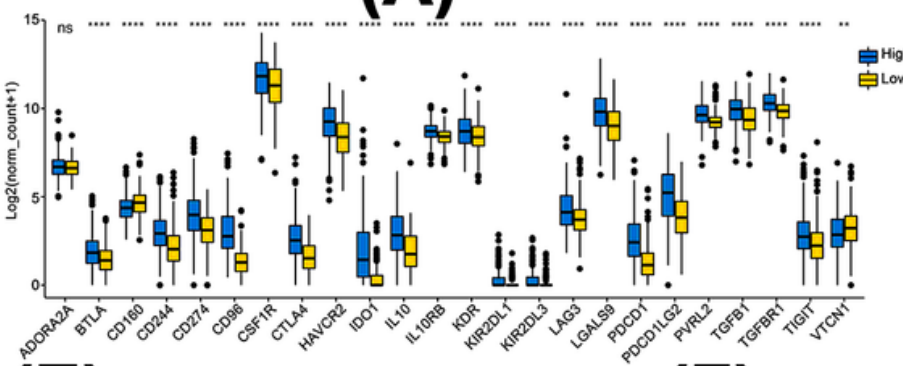

(E) TIDE
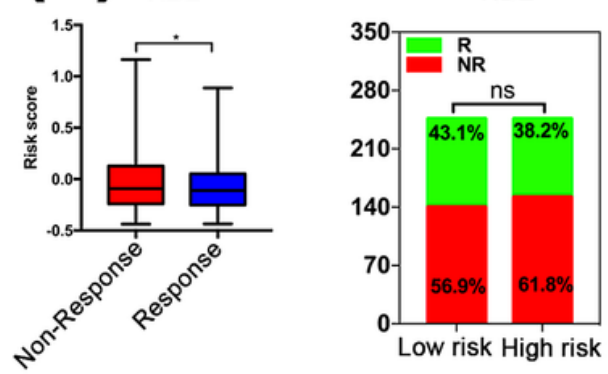

(F)

ImmuCellAI

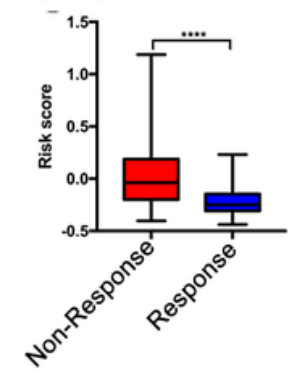

(H)
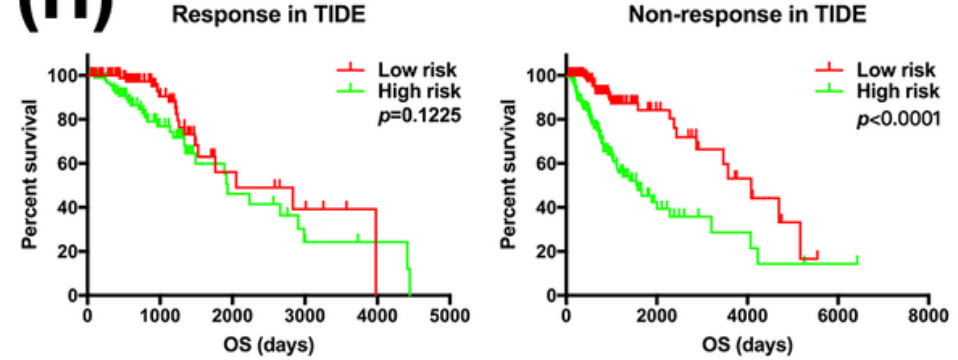

(B)
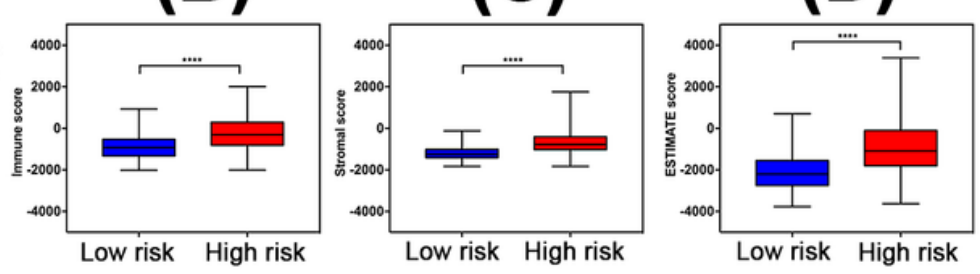

(G)
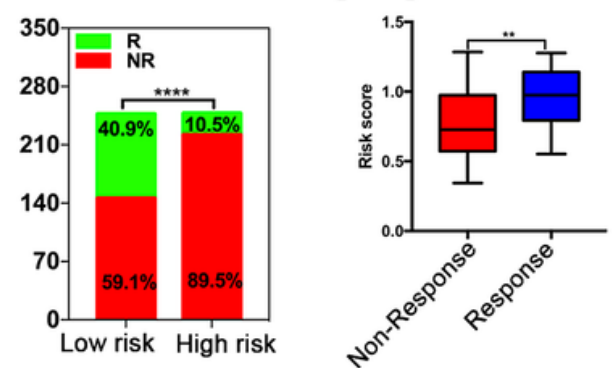

GSE35640

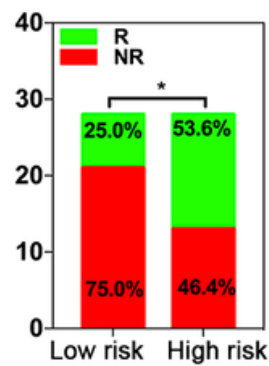

Low risk High risk
(D)
(I)

Response in ImmuCellAI

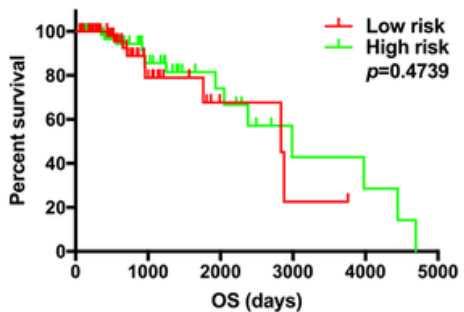

\section{Figure 10}

LGG patients with high-risk scores present immune checkpoints blockade (ICB) treatment resistance. A High-risk scores presented high expression of PDCD1, CD274, CTLA-4 and LAG-3 and other immunoinhibitory molecules. B-D Through ESTIMATE algorithm, we found that patients in the high-risk score group have higher immune (B), stromal (C) and ESTIMATE (D) scores. (E-F) Using the TIDE (E) and ImmuCellAI (F) methods, we found "non-response" group patients have higher risk scores. G High-risk scores in the GSE35640 dataset showed better effects on ICB treatment. (H-I) Based on the median risk scores, the LGG patients with high-risk scores in the "non-response" groups had a shorter OS, but no significance was found in the "response" groups. *: $p<0.05 ; * \star: p<0.01 ; * \star *: p<0.001 ; * \star \star *: p<0.0001$

\section{Supplementary Files}

This is a list of supplementary files associated with this preprint. Click to download.

- FigS1.tif

- Figs2.tif

- FigS3.tif

- TableS1.doc

- Tables2.doc

- TableS3.doc

- Tables4.doc 
- TableS5.doc

- TableS6.doc

- Tables7.doc 